\title{
Systematic Evaluation of ReaxFF Reactive Force Fields for Biochemical Applications
}

\author{
Evgeny Moerman, ${ }^{\dagger}$ David Furman, ${ }^{*,+, \dagger}$ and David J. Wales ${ }^{*, \dagger}$ \\ $\dagger$ Yusuf Hamied Department of Chemistry, University of Cambridge, Lensfield \\ Road,Cambridge CB2 1EW, United Kingdom \\ $\ddagger$ Division of Chemistry, NRCN, P.O. Box 9001, Beer-Sheva 84190, Israel \\ E-mail: df398@cam.ac.uk; dw34@cam.ac.uk
}




\section{Molecular geometries}

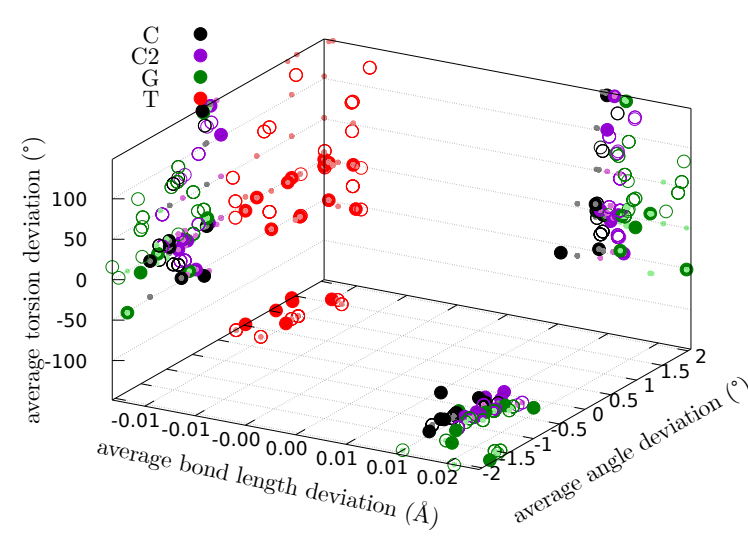

(a) glycine

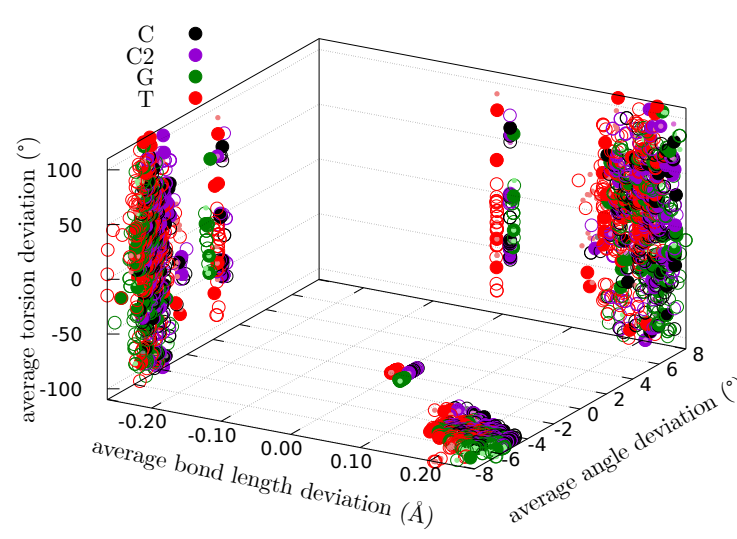

(c) tryptophane

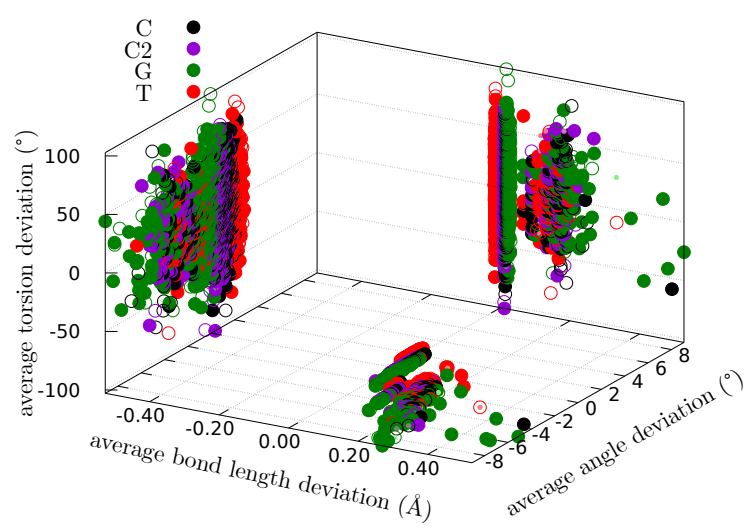

(e) arginine

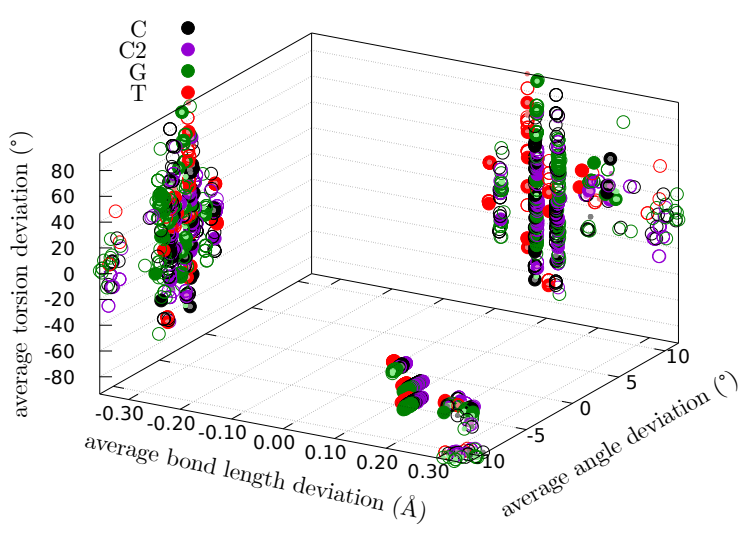

(b) valine

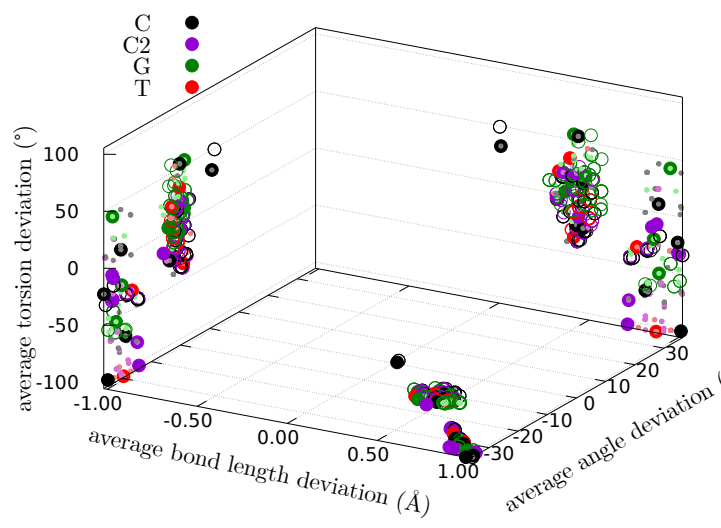

(d) alanine

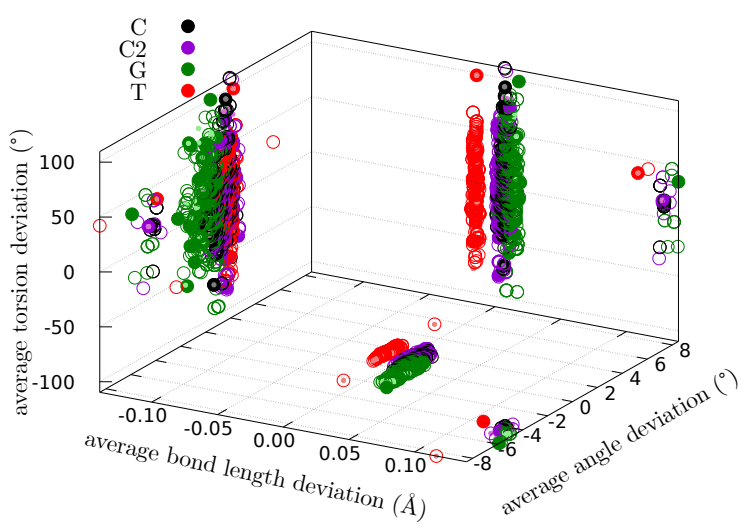

(f) asparagine 


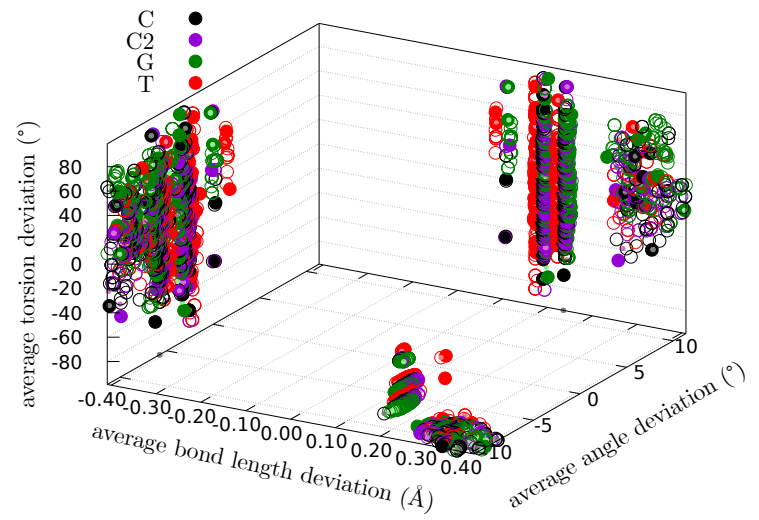

(g) aspartic acid

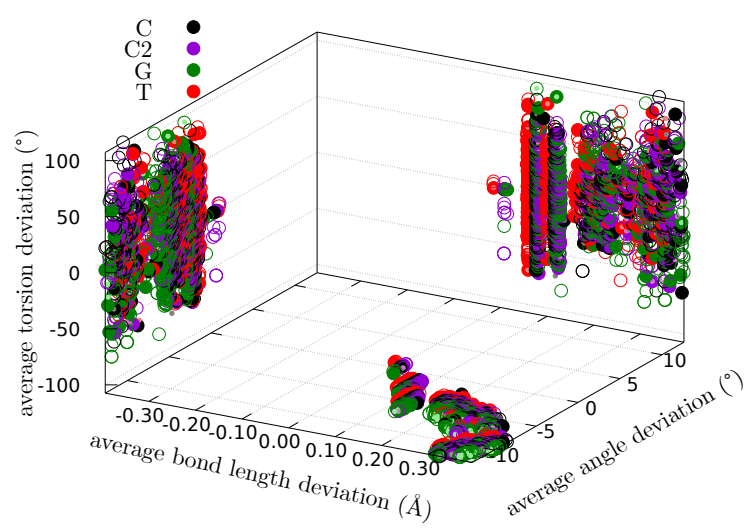

(i) glutamic acid

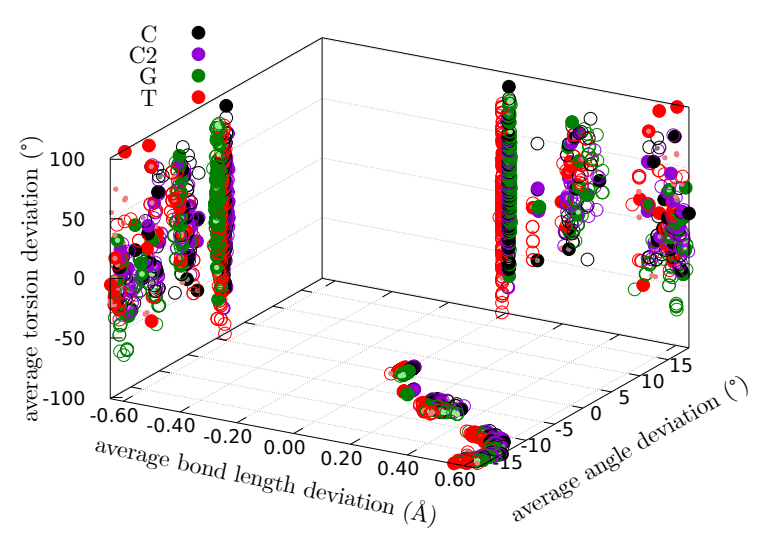

(k) histidine

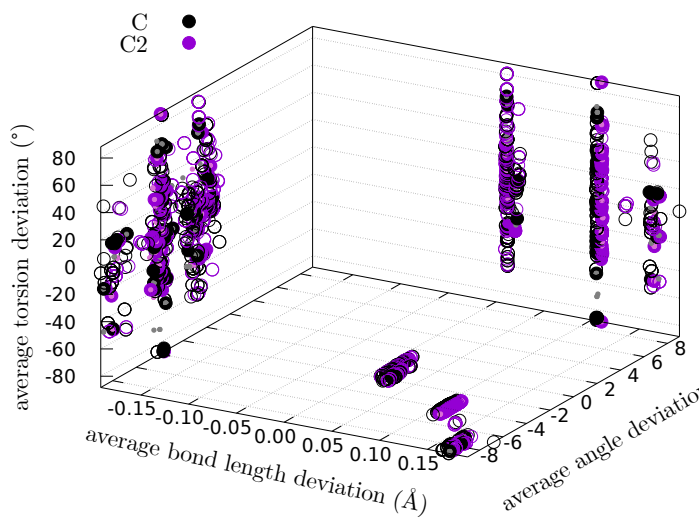

(h) cysteine

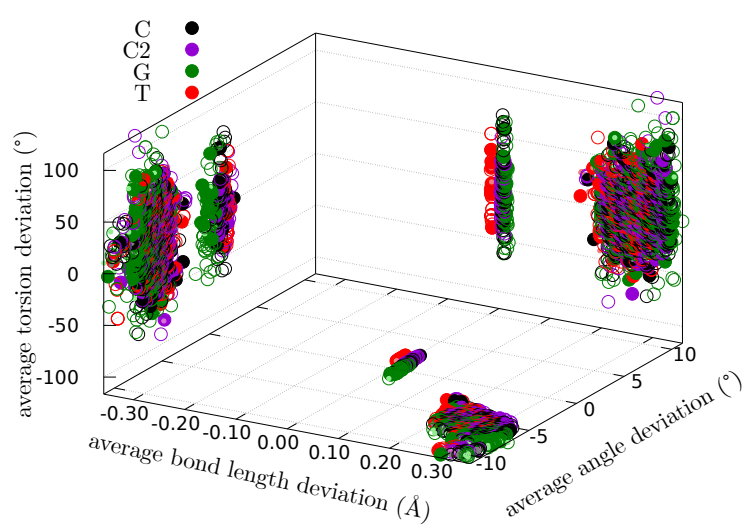

(j) glutamine

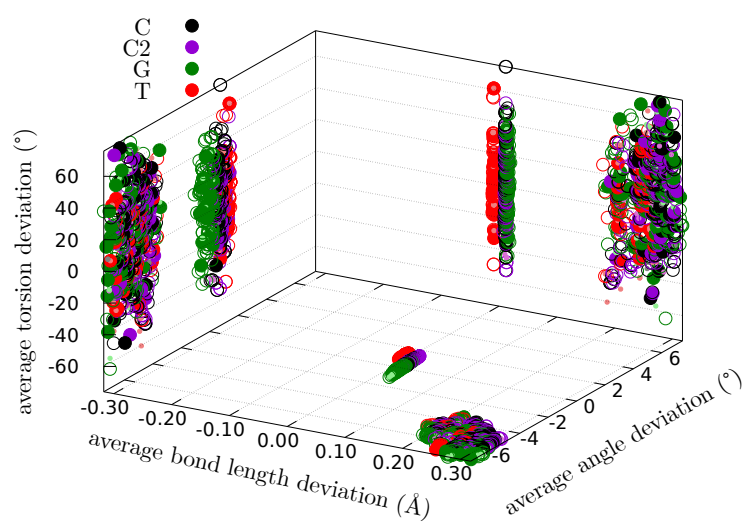

(1) isoleucine 


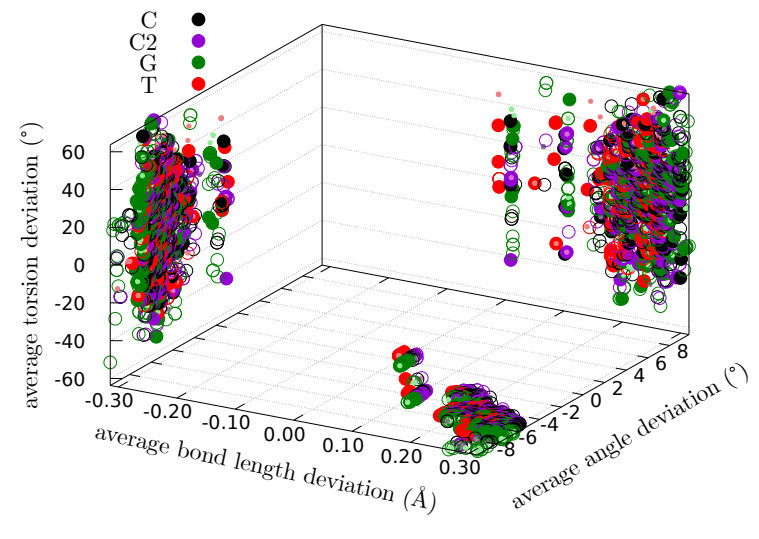

(m) leucine

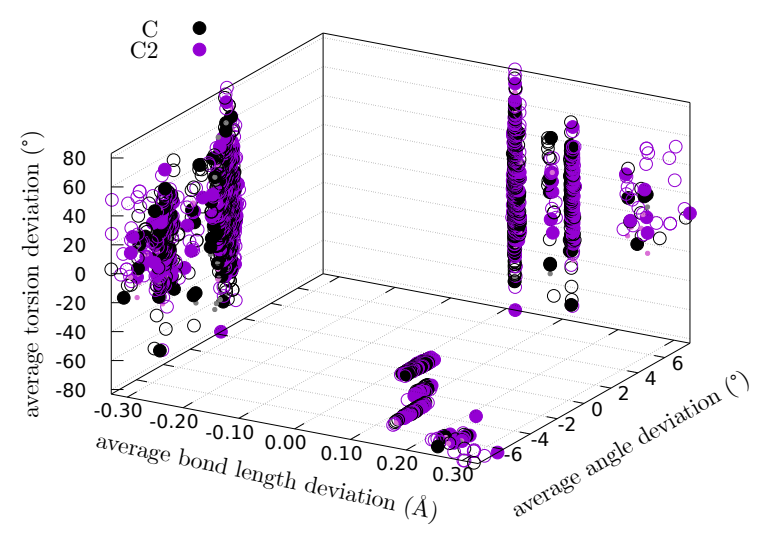

(o) methionine

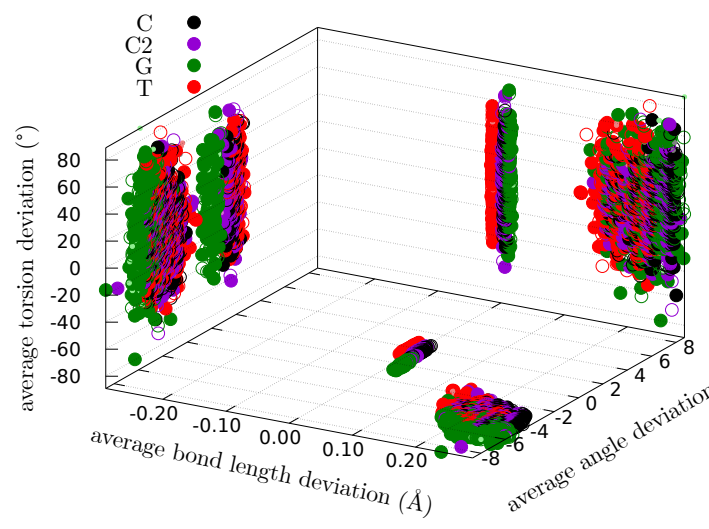

(n) lysine

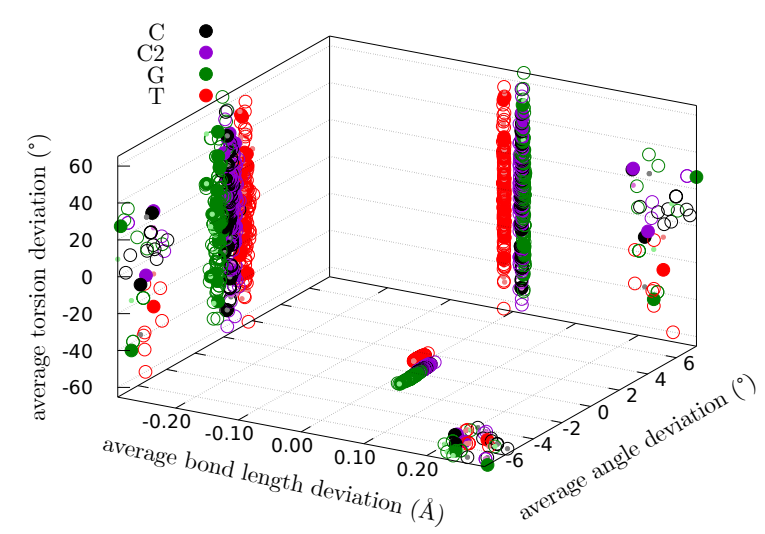

(p) phenylalanine 


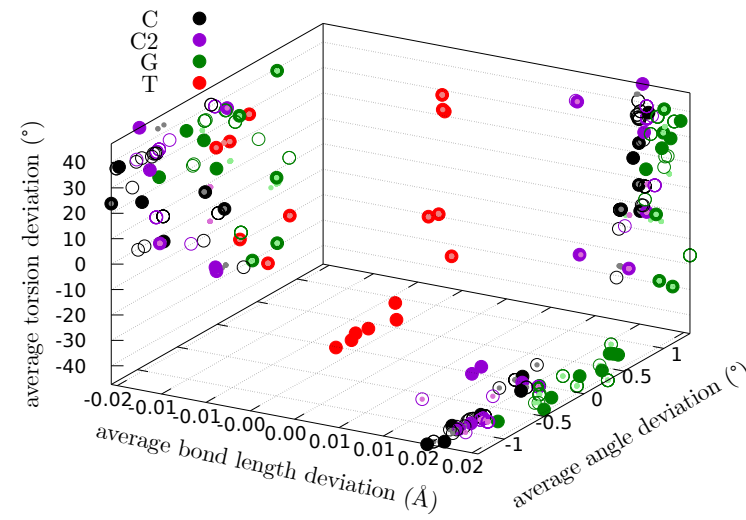

(q) proline

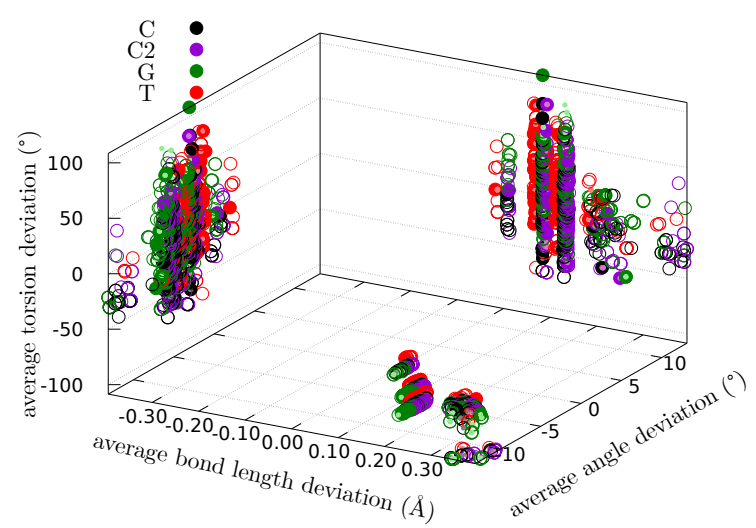

(s) threonine

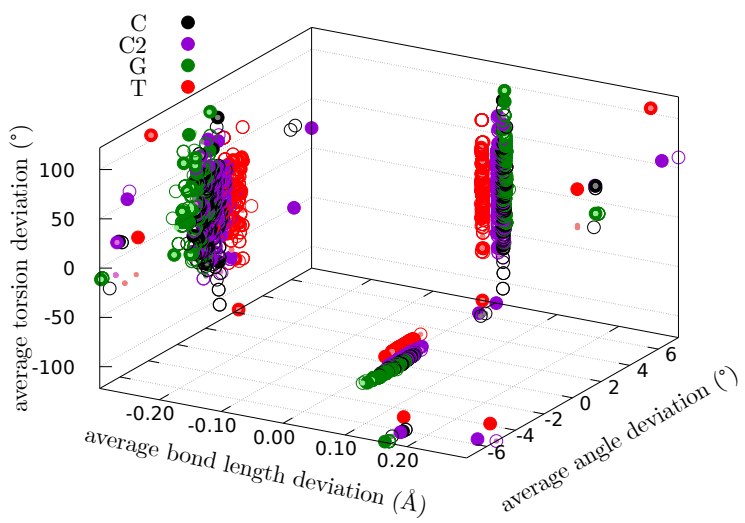

(r) serine

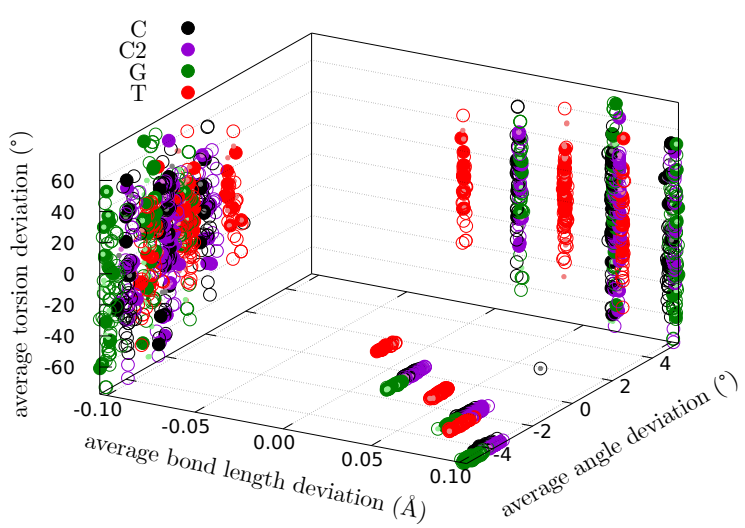

(t) tyrosine

Figure S1: Average deviation of internal coordinates for conformers of Glycine, Valine and Tryptophane with different ReaxFF force fields compared to QM reference structures. Filled circles: physical conformers, empty circles: artificial conformers and dots: artificial noise.

\section{Relative stability of conformers}

The $\mathbf{M}$ values provided for the plots are a metric for the quality of their monotonously rising behaviour. The values were obtained by applying a "moving average" algorithm to the data points of every plot: If the complete data set of a plot already featured perfectly monotonic behaviour, it was assigned a $\mathbf{M}$ value equal to the smallest QM energy difference between two adjacent points. If the most left and right data point themselves did not align monotonically, $\mathbf{M}$ was set equal to the complete QM energy range. Otherwise an iterative algorithm was 
performed, which shall be explained on the example of Tryptophane using force field $\mathbf{C}$ : An initial $\mathbf{M}_{\mathbf{0}}$ was set to half of the complete $\mathrm{QM}$ range, here $4.03 \mathrm{kcal} / \mathrm{mol}$. The range of the plot was then separated into a region of $0 \mathrm{kcal} / \mathrm{mol}<\mathrm{QM}$ energy $<4.03 \mathrm{kcal} / \mathrm{mol}$ and 4.03 $\mathrm{kcal} / \mathrm{mol}<\mathrm{QM}$ energy $<8.06 \mathrm{kcal} / \mathrm{mol}$. For both regions the average force field energy of the therein contained data points was determined, here $1.68 \mathrm{kcal} / \mathrm{mol}$ for the first region and $2.01 \mathrm{kcal} / \mathrm{mol}$ for the second one. If the obtained averaged data points describe a monotonic behaviour, as it would be the case in this example, $\mathbf{M}_{\mathbf{1}}$ is set to the average between the current $\mathbf{M}\left(=\mathbf{M}_{\mathbf{0}}\right)$ value and the minimum range value, initially $0 \mathrm{kcal} / \mathrm{mol}$. Otherwise, $\mathbf{M}_{\mathbf{1}}$ is chosen as the average between the current $\mathbf{M}$ value and the maximum range value, initially the QM energy maximum. This algorithm was performed until the $\mathbf{M}$ value didn't change by more than $0.10 \mathrm{kcal} / \mathrm{mol}$. The $\mathbf{M}$ obtained in this way states how local the monotonic behaviour of a data set is. The lower $\mathbf{M}$ is, the more fine-grained is the monotonic behaviour. The second metric shown in figure S2 is the resolution $\mathbf{R}$. It reflects the minimum QM energy difference of two conformers above which the force field correctly predicts the energetic ordering. $\mathbf{R}$ is obtained by dividing the plotted data into two bins based on a value $x$ : The two bins contain the points with $0<\mathrm{QM}$ energy $<x$ and $\mathrm{E}_{\mathrm{QM} \text {,max }}-x<\mathrm{QM}$ energy $<$ $\mathrm{E}_{\mathrm{QM}, \max }, \mathrm{E}_{\mathrm{QM}, \max }$ being the maximum $\mathrm{QM}$ energy in the data set. Therefore, initially only a subset of all data points is included. If the data points of the two bins feature a monotonic behaviour, the bins are widened by increasing $x$ by the minimal QM energy difference of two adjacent data points in the complete data set. This is done iteratively until monotony does not hold anymore. By taking the difference between the complete QM energy range and twice the final width $x$ of the bins, one obtains the minimal energy difference between two conformers i.e data points, above which monotonic ordering is ensured. 


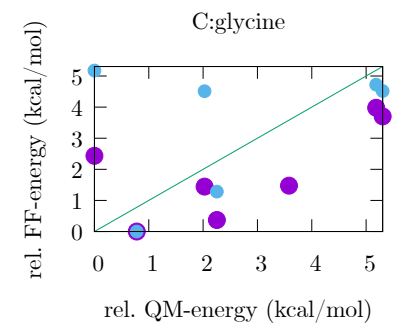

$\mathrm{M}=2.24 \mathrm{kcal} / \mathrm{mol}$

$\mathrm{R}=5.06 \mathrm{kcal} / \mathrm{mol}$

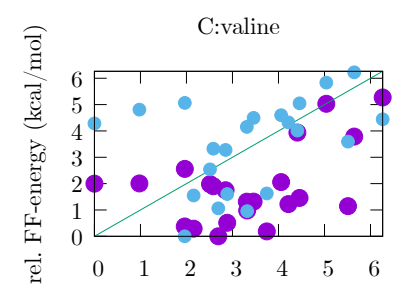

rel. QM-energy (kcal/mol)

$\mathrm{M}=2.45 \mathrm{kcal} / \mathrm{mol}$

$\mathrm{R}=4.76 \mathrm{kcal} / \mathrm{mol}$

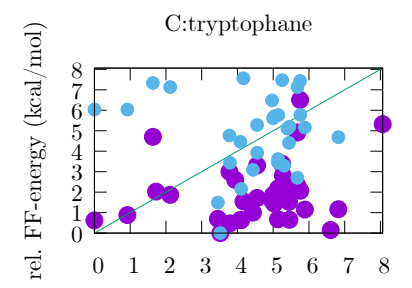

rel. QM-energy (kcal/mol)

$\mathrm{M}=3.46 \mathrm{kcal} / \mathrm{mol}$ $\mathrm{R}=5.15 \mathrm{kcal} / \mathrm{mol}$

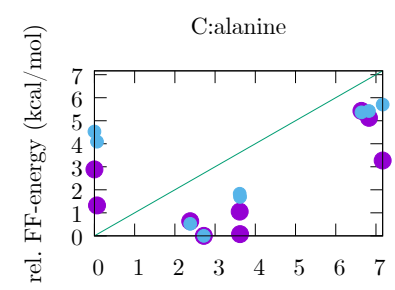

rel. QM-energy (kcal/mol)

$\mathrm{M}=3.30 \mathrm{kcal} / \mathrm{mol}$

$\mathrm{R}=7.05 \mathrm{kcal} / \mathrm{mol}$

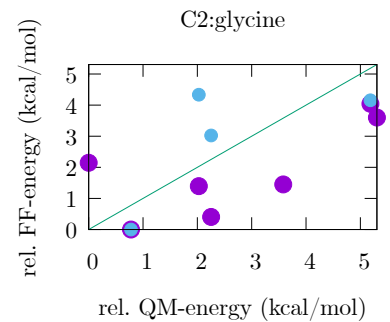

$\mathrm{M}=1.74 \mathrm{kcal} / \mathrm{mol}$

$\mathrm{R}=5.06 \mathrm{kcal} / \mathrm{mol}$

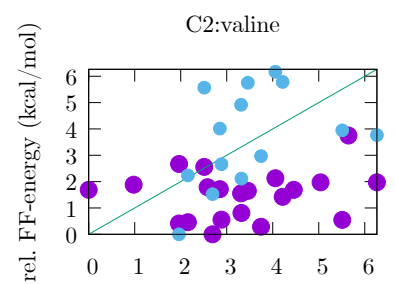

rel. QM-energy (kcal/mol)

$\mathrm{M}=2.84 \mathrm{kcal} / \mathrm{mol}$

$\mathrm{R}=5.03 \mathrm{kcal} / \mathrm{mol}$

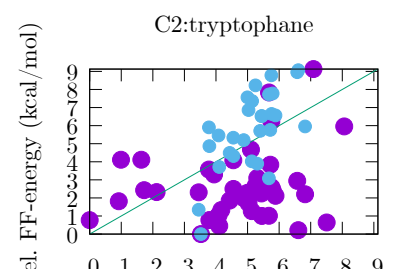

rel. QM-energy (kcal/mol)

$\mathrm{M}=3.46 \mathrm{kcal} / \mathrm{mol}$

$\mathrm{R}=6.95 \mathrm{kcal} / \mathrm{mol}$

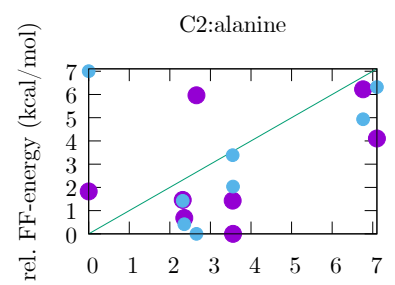

rel. QM-energy (kcal/mol)

$\mathrm{M}=3.38 \mathrm{kcal} / \mathrm{mol}$

$\mathrm{R}=6.43 \mathrm{kcal} / \mathrm{mol}$

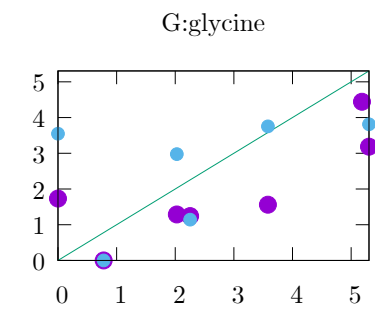

$\mathrm{M}=0.75 \mathrm{kcal} / \mathrm{mol}$

$\mathrm{R}=5.06 \mathrm{kcal} / \mathrm{mol}$

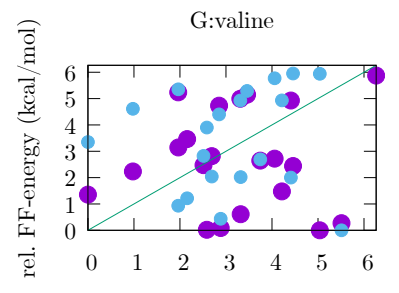

rel. QM-energy (kcal/mol)

$\mathrm{M}=4.99 \mathrm{kcal} / \mathrm{mol}$

$\mathrm{R}=5.76 \mathrm{kcal} / \mathrm{mol}$

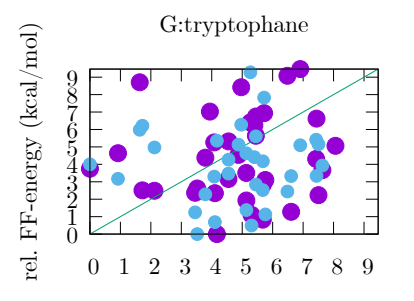

rel. QM-energy (kcal/mol)

$\mathrm{M}=3.84 \mathrm{kcal} / \mathrm{mol}$

$\mathrm{R}=7.20 \mathrm{kcal} / \mathrm{mol}$

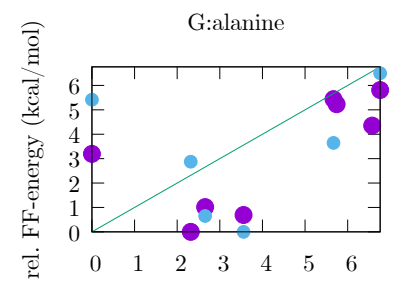

rel. QM-energy (kcal/mol)

$\mathrm{M}=2.79 \mathrm{kcal} / \mathrm{mol}$

$\mathrm{R}=4.72 \mathrm{kcal} / \mathrm{mol}$

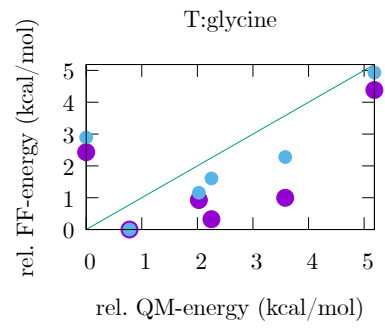

$\mathrm{M}=2.18 \mathrm{kcal} / \mathrm{mol}$

$\mathrm{R}=3.83 \mathrm{kcal} / \mathrm{mol}$

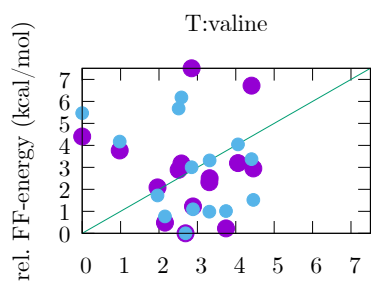

rel. QM-energy (kcal/mol)

$\mathrm{M}=4.46 \mathrm{kcal} / \mathrm{mol}$

$\mathrm{R}=4.46 \mathrm{kcal} / \mathrm{mol}$

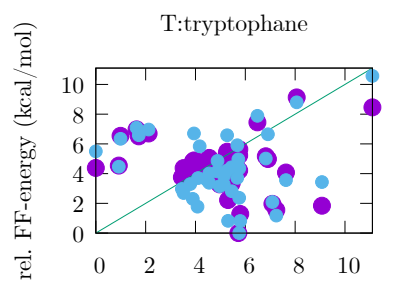

rel. QM-energy (kcal/mol)

$\mathrm{M}=7.02 \mathrm{kcal} / \mathrm{mol}$

$\mathrm{R}=7.65 \mathrm{kcal} / \mathrm{mol}$

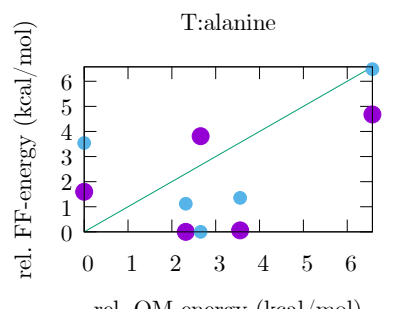

$\mathrm{M}=3.34 \mathrm{kcal} / \mathrm{mol}$

$\mathrm{R}=2.51 \mathrm{kcal} / \mathrm{mol}$ 


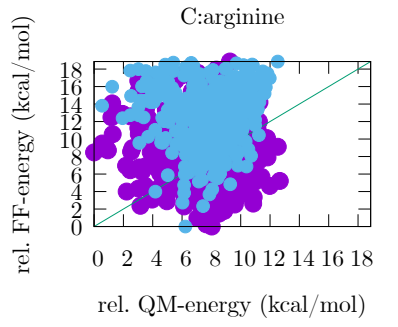

$\mathrm{M}=12.67 \mathrm{kcal} / \mathrm{mol}$

$\mathrm{R}=12.67 \mathrm{kcal} / \mathrm{mol}$

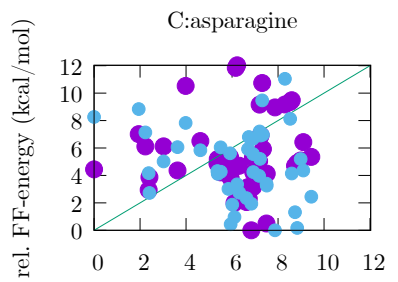

rel. QM-energy (kcal/mol)

$\mathrm{M}=5.53 \mathrm{kcal} / \mathrm{mol}$

$\mathrm{R}=8.76 \mathrm{kcal} / \mathrm{mol}$

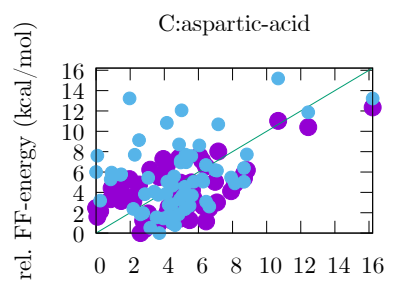

rel. QM-energy (kcal/mol)

$\mathrm{M}=3.10 \mathrm{kcal} / \mathrm{mol}$ $\mathrm{R}=16.06 \mathrm{kcal} / \mathrm{mol}$

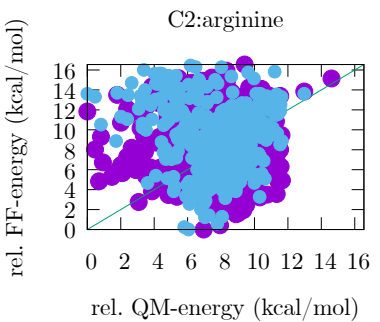

$\mathrm{M}=11.36 \mathrm{kcal} / \mathrm{mol}$

$\mathrm{R}=13.69 \mathrm{kcal} / \mathrm{mol}$

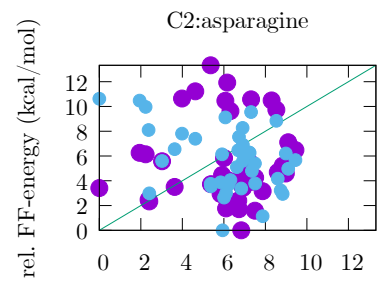

rel. QM-energy (kcal/mol)

$\mathrm{M}=6.86 \mathrm{kcal} / \mathrm{mol}$

$\mathrm{R}=8.75 \mathrm{kcal} / \mathrm{mol}$

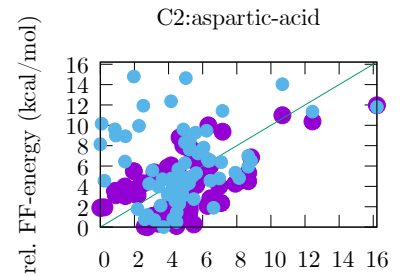

rel. QM-energy (kcal/mol)

$\mathrm{M}=3.10 \mathrm{kcal} / \mathrm{mol}$

$\mathrm{R}=14.39 \mathrm{kcal} / \mathrm{mol}$

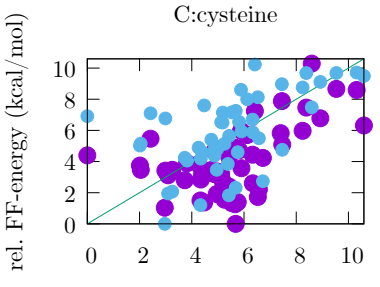

rel. QM-energy (kcal/mol)

$\mathrm{M}=4.05 \mathrm{kcal} / \mathrm{mol}$

$\mathrm{R}=10.04 \mathrm{kcal} / \mathrm{mol}$
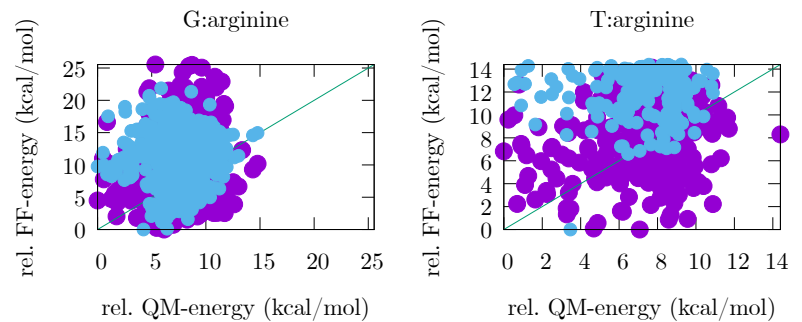

$\mathrm{M}=6.29 \mathrm{kcal} / \mathrm{mol}$

$\mathrm{M}=7.14 \mathrm{kcal} / \mathrm{mol}$

$\mathrm{R}=13.79 \mathrm{kcal} / \mathrm{mol}$

$\mathrm{R}=13.92 \mathrm{kcal} / \mathrm{mol}$

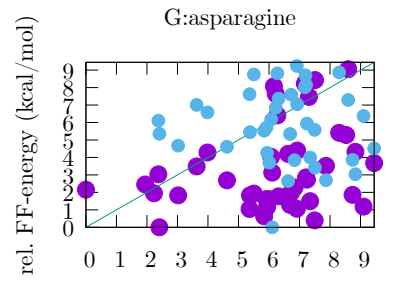

rel. QM-energy (kcal/mol)

$\mathrm{M}=1.99 \mathrm{kcal} / \mathrm{mol}$

$\mathrm{R}=8.76 \mathrm{kcal} / \mathrm{mol}$

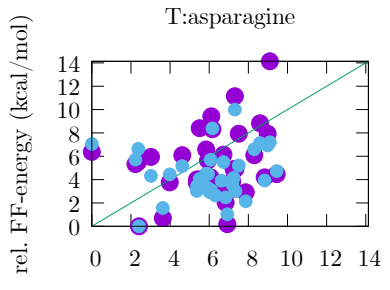

rel. QM-energy (kcal/mol)

$\mathrm{M}=9.44 \mathrm{kcal} / \mathrm{mol}$

$\mathrm{R}=9.44 \mathrm{kcal} / \mathrm{mol}$

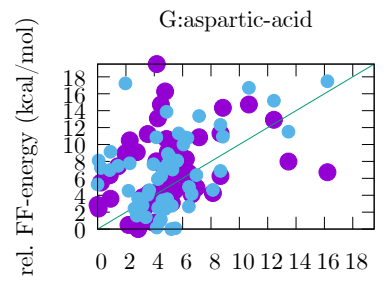

rel. QM-energy (kcal/mol)

$\mathrm{M}=3.99 \mathrm{kcal} / \mathrm{mol}$

$\mathrm{R}=16.06 \mathrm{kcal} / \mathrm{mol}$

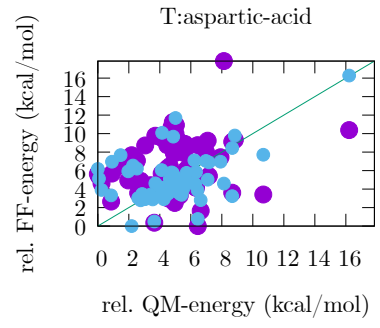

$\mathrm{M}=3.61 \mathrm{kcal} / \mathrm{mol}$

$\mathrm{R}=16.06 \mathrm{kcal} / \mathrm{mol}$

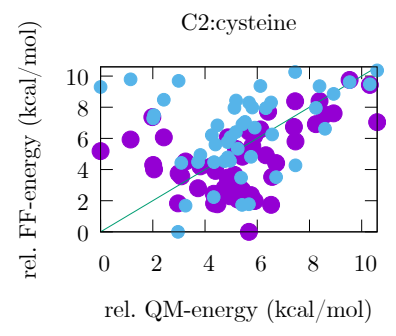

$\mathrm{M}=4.38 \mathrm{kcal} / \mathrm{mol}$

$\mathrm{R}=10.04 \mathrm{kcal} / \mathrm{mol}$ 


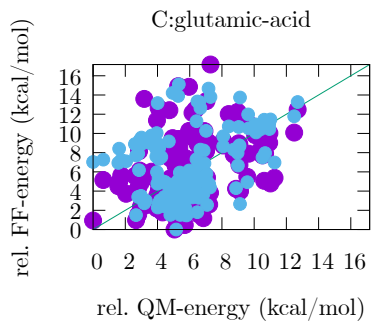

$\mathrm{M}=2.68 \mathrm{kcal} / \mathrm{mol}$

$\mathrm{R}=9.56 \mathrm{kcal} / \mathrm{mol}$

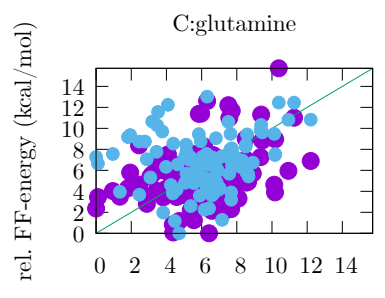

rel. QM-energy (kcal/mol)

$\mathrm{M}=2.95 \mathrm{kcal} / \mathrm{mol}$

$\mathrm{R}=10.30 \mathrm{kcal} / \mathrm{mol}$

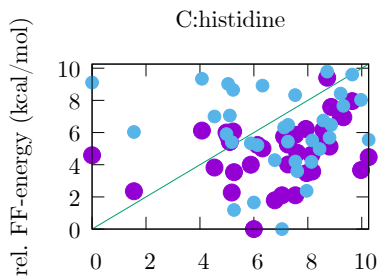

rel. QM-energy (kcal/mol)

$\mathrm{M}=10.25 \mathrm{kcal} / \mathrm{mol}$

$\mathrm{R}=10.25 \mathrm{kcal} / \mathrm{mol}$

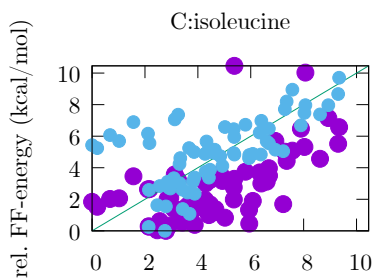

rel. QM-energy $(\mathrm{kcal} / \mathrm{mol})$

$\mathrm{M}=2.70 \mathrm{kcal} / \mathrm{mol}$

$\mathrm{R}=8.95 \mathrm{kcal} / \mathrm{mol}$

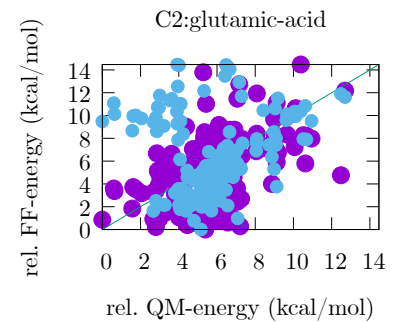

$\mathrm{M}=2.48 \mathrm{kcal} / \mathrm{mol}$

$\mathrm{R}=11.43 \mathrm{kcal} / \mathrm{mol}$

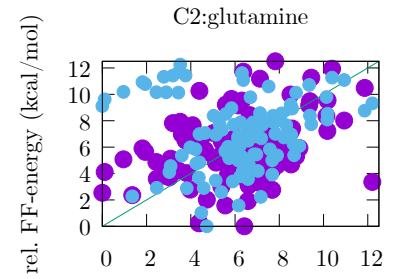

rel. QM-energy (kcal/mol)

$\mathrm{M}=2.95 \mathrm{kcal} / \mathrm{mol}$

$\mathrm{R}=11.99 \mathrm{kcal} / \mathrm{mol}$

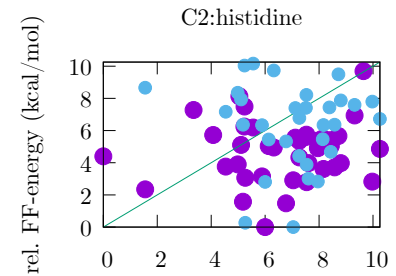

rel. QM-energy (kcal/mol)

$\mathrm{M}=6.01 \mathrm{kcal} / \mathrm{mol}$

$\mathrm{R}=9.69 \mathrm{kcal} / \mathrm{mol}$

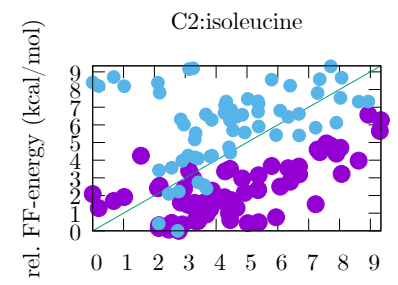

rel. QM-energy (kcal/mol)

$\mathrm{M}=2.70 \mathrm{kcal} / \mathrm{mol}$

$\mathrm{R}=8.95 \mathrm{kcal} / \mathrm{mol}$

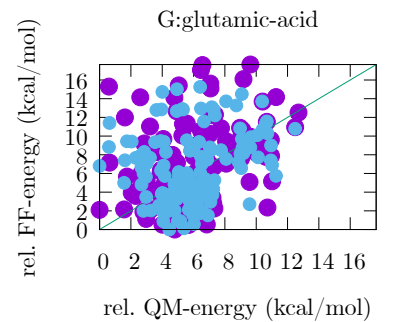

$\mathrm{M}=4.07 \mathrm{kcal} / \mathrm{mol}$

$\mathrm{R}=11.50 \mathrm{kcal} / \mathrm{mol}$

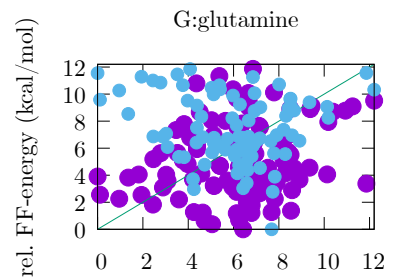

rel. QM-energy (kcal/mol)

$\mathrm{M}=4.67 \mathrm{kcal} / \mathrm{mol}$

$\mathrm{R}=11.99 \mathrm{kcal} / \mathrm{mol}$

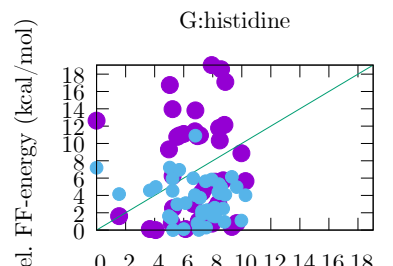

rel. QM-energy (kcal/mol)

$\mathrm{M}=10.26 \mathrm{kcal} / \mathrm{mol}$

$\mathrm{R}=10.25 \mathrm{kcal} / \mathrm{mol}$

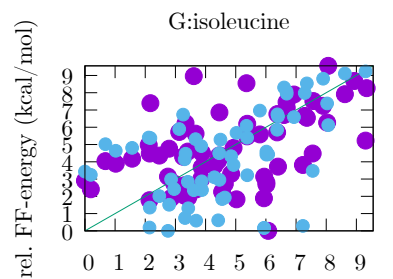

rel. QM-energy (kcal/mol)

$\mathrm{M}=1.39 \mathrm{kcal} / \mathrm{mol}$

$\mathrm{R}=8.95 \mathrm{kcal} / \mathrm{mol}$

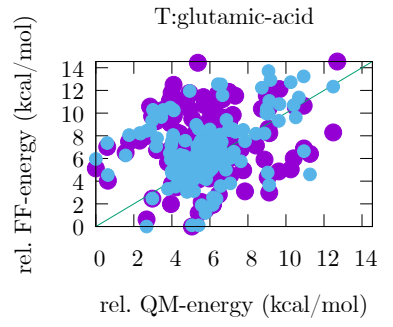

$\mathrm{M}=2.88 \mathrm{kcal} / \mathrm{mol}$

$\mathrm{R}=11.43 \mathrm{kcal} / \mathrm{mol}$

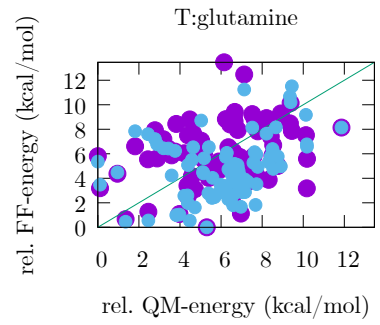

$\mathrm{M}=2.69 \mathrm{kcal} / \mathrm{mol}$

$\mathrm{R}=11.65 \mathrm{kcal} / \mathrm{mol}$

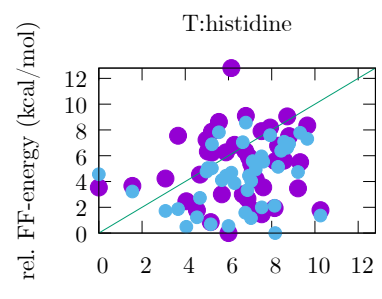

rel. QM-energy (kcal/mol)

$\mathrm{M}=10.26 \mathrm{kcal} / \mathrm{mol}$

$\mathrm{R}=10.25 \mathrm{kcal} / \mathrm{mol}$

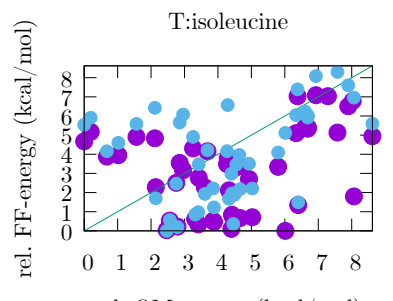

$\mathrm{M}=3.43 \mathrm{kcal} / \mathrm{mol}$

$\mathrm{R}=8.23 \mathrm{kcal} / \mathrm{mol}$ 


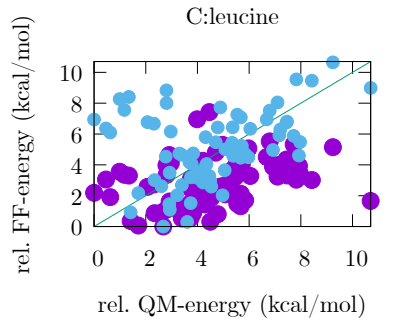

$\mathrm{M}=10.70 \mathrm{kcal} / \mathrm{mol}$

$\mathrm{R}=10.70 \mathrm{kcal} / \mathrm{mol}$

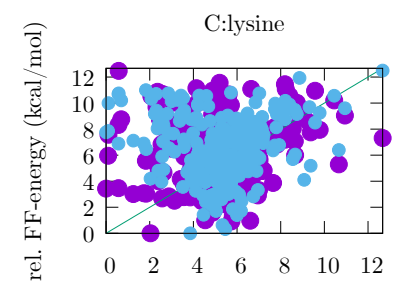

rel. QM-energy (kcal/mol)

$\mathrm{M}=4.45 \mathrm{kcal} / \mathrm{mol}$

$\mathrm{R}=12.45 \mathrm{kcal} / \mathrm{mol}$

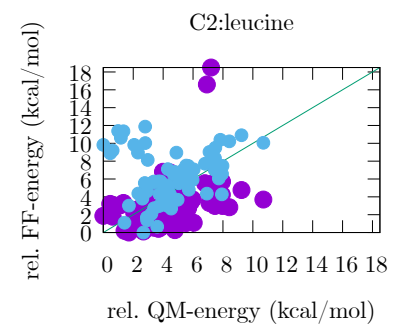

$\mathrm{M}=5.27 \mathrm{kcal} / \mathrm{mol}$

$\mathrm{R}=9.46 \mathrm{kcal} / \mathrm{mol}$

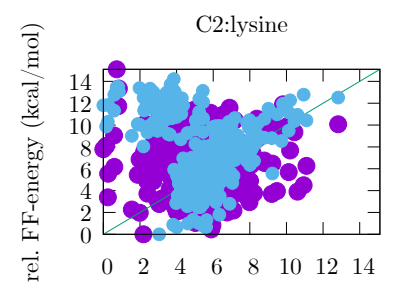

rel. QM-energy (kcal/mol)

$\mathrm{M}=5.96 \mathrm{kcal} / \mathrm{mol}$

$\mathrm{R}=12.33 \mathrm{kcal} / \mathrm{mol}$

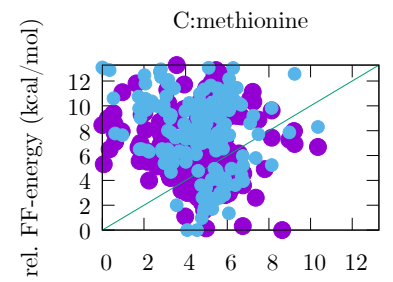

rel. QM-energy (kcal/mol)

$\mathrm{M}=10.35 \mathrm{kcal} / \mathrm{mol}$

$\mathrm{R}=10.35 \mathrm{kcal} / \mathrm{mol}$

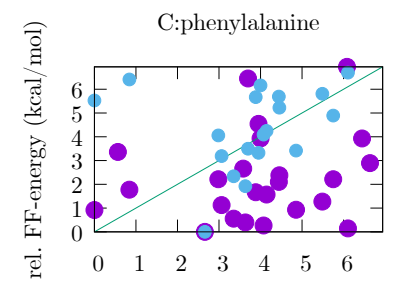

rel. QM-energy (kcal/mol)

$\mathrm{M}=2.02 \mathrm{kcal} / \mathrm{mol}$

$\mathrm{R}=6.26 \mathrm{kcal} / \mathrm{mol}$

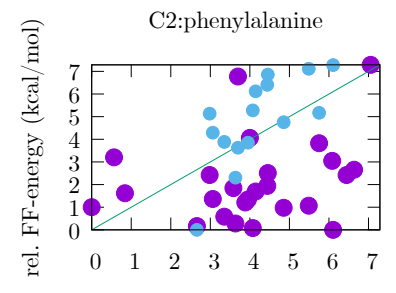

rel. QM-energy (kcal/mol)

$\mathrm{M}=2.70 \mathrm{kcal} / \mathrm{mol}$

$\mathrm{R}=5.93 \mathrm{kcal} / \mathrm{mol}$

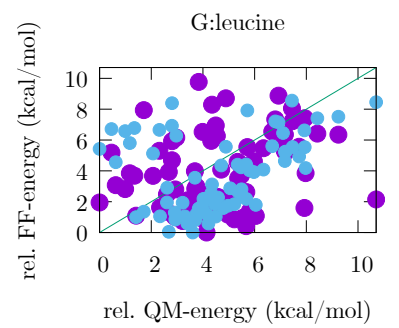

$\mathrm{M}=2.59 \mathrm{kcal} / \mathrm{mol}$

$\mathrm{R}=9.78 \mathrm{kcal} / \mathrm{mol}$

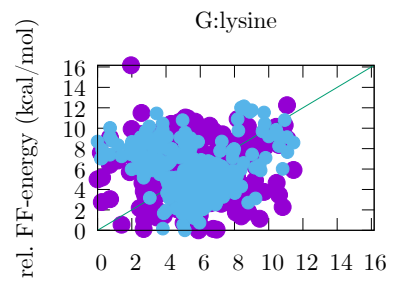

rel. QM-energy (kcal/mol)

$\mathrm{M}=5.99 \mathrm{kcal} / \mathrm{mol}$

$\mathrm{R}=11.07 \mathrm{kcal} / \mathrm{mol}$

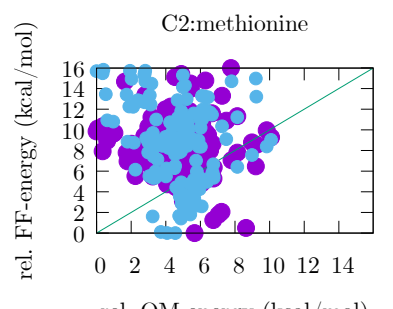

$\mathrm{M}=10.08 \mathrm{kcal} / \mathrm{mol}$

$\mathrm{R}=10.08 \mathrm{kcal} / \mathrm{mol}$

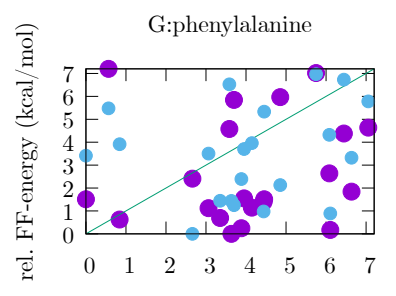

rel. QM-energy (kcal/mol)

$\mathrm{M}=3.25 \mathrm{kcal} / \mathrm{mol}$

$\mathrm{R}=5.93 \mathrm{kcal} / \mathrm{mol}$

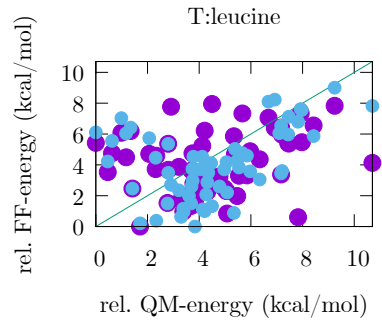

$\mathrm{M}=10.70 \mathrm{kcal} / \mathrm{mol}$

$\mathrm{R}=10.70 \mathrm{kcal} / \mathrm{mol}$

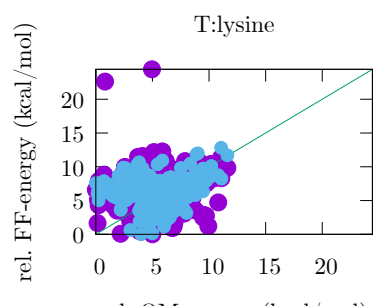

$\mathrm{M}=6.63 \mathrm{kcal} / \mathrm{mol}$

$\mathrm{R}=11.31 \mathrm{kcal} / \mathrm{mol}$

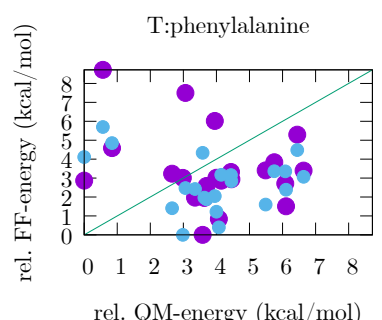

$\mathrm{M}=6.06 \mathrm{kcal} / \mathrm{mol}$

$\mathrm{R}=6.26 \mathrm{kcal} / \mathrm{mol}$ 


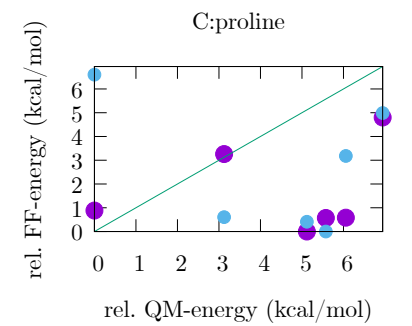

$\mathrm{M}=5.15 \mathrm{kcal} / \mathrm{mol}$

$\mathrm{R}=6.03 \mathrm{kcal} / \mathrm{mol}$

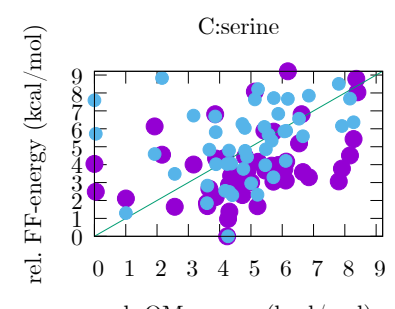

rel. QM-energy (kcal/mol)

$\mathrm{M}=3.09 \mathrm{kcal} / \mathrm{mol}$

$\mathrm{R}=8.33 \mathrm{kcal} / \mathrm{mol}$

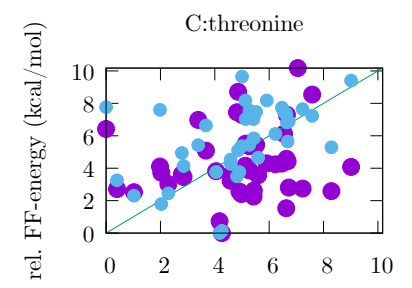

rel. QM-energy (kcal/mol)

$\mathrm{M}=9.01 \mathrm{kcal} / \mathrm{mol}$

$\mathrm{R}=9.01 \mathrm{kcal} / \mathrm{mol}$

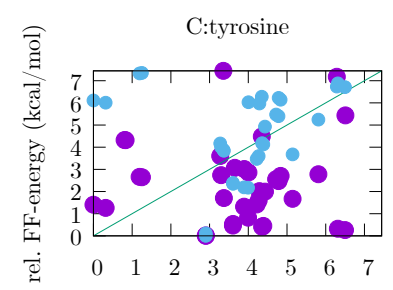

rel. QM-energy (kcal/mol)

$\mathrm{M}=2.89 \mathrm{kcal} / \mathrm{mol}$

$\mathrm{R}=6.49 \mathrm{kcal} / \mathrm{mol}$

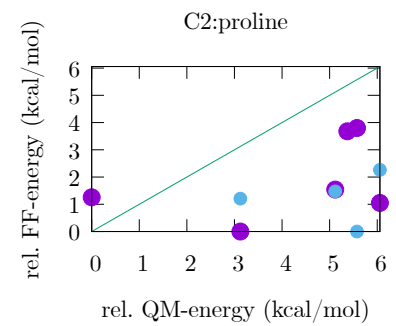

$\mathrm{M}=6.06 \mathrm{kcal} / \mathrm{mol}$

$\mathrm{R}=6.06 \mathrm{kcal} / \mathrm{mol}$

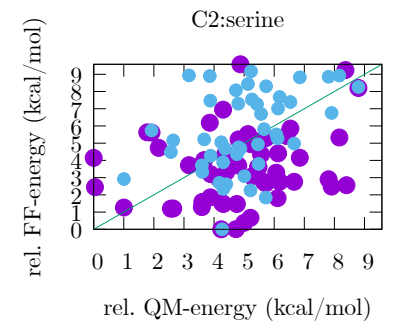

$\mathrm{M}=2.68 \mathrm{kcal} / \mathrm{mol}$

$\mathrm{R}=8.73 \mathrm{kcal} / \mathrm{mol}$

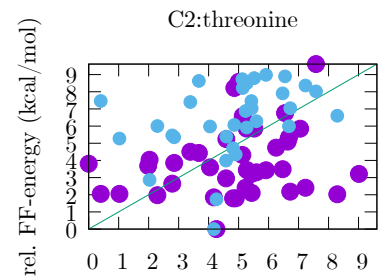

rel. QM-energy (kcal/mol)

$\mathrm{M}=9.01 \mathrm{kcal} / \mathrm{mol}$

$\mathrm{R}=9.01 \mathrm{kcal} / \mathrm{mol}$

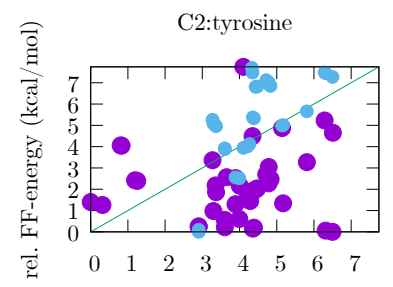

rel. QM-energy (kcal/mol)

$\mathrm{M}=2.89 \mathrm{kcal} / \mathrm{mol}$

$\mathrm{R}=6.49 \mathrm{kcal} / \mathrm{mol}$

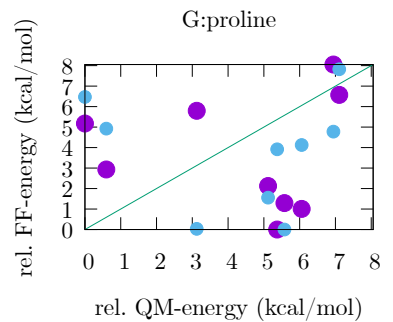

$\mathrm{M}=5.38 \mathrm{kcal} / \mathrm{mol}$

$\mathrm{R}=6.78 \mathrm{kcal} / \mathrm{mol}$

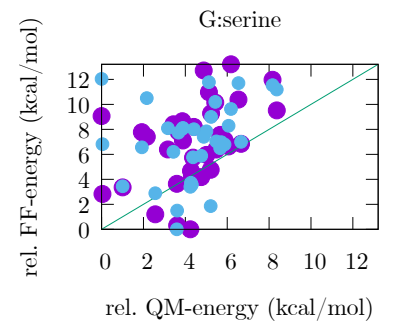

$\mathrm{M}=2.55 \mathrm{kcal} / \mathrm{mol}$

$\mathrm{R}=8.31 \mathrm{kcal} / \mathrm{mol}$

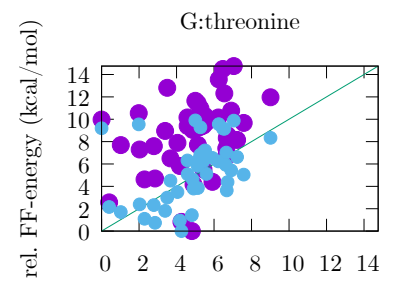

rel. QM-energy (kcal/mol)

\section{$\mathrm{M}=2.60 \mathrm{kcal} / \mathrm{mol}$}

$\mathrm{R}=8.21 \mathrm{kcal} / \mathrm{mol}$

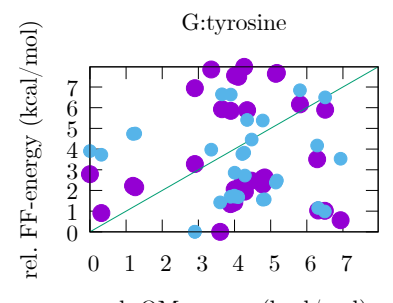

rel. QM-energy (kcal/mol)

$\mathrm{M}=6.93 \mathrm{kcal} / \mathrm{mol}$

$\mathrm{R}=6.93 \mathrm{kcal} / \mathrm{mol}$

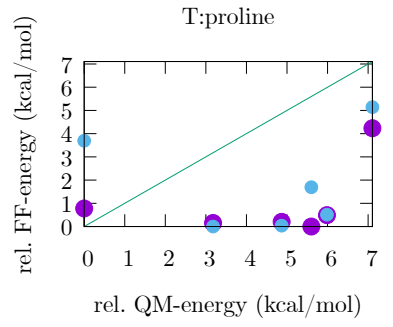

$\mathrm{M}=3.61 \mathrm{kcal} / \mathrm{mol}$

$\mathrm{R}=5.56 \mathrm{kcal} / \mathrm{mol}$

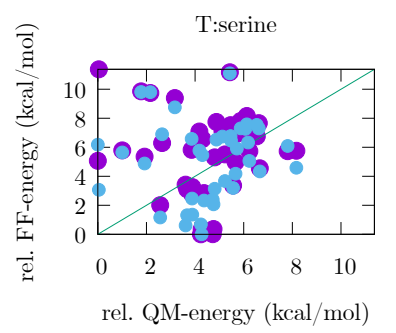

$\mathrm{M}=4.27 \mathrm{kcal} / \mathrm{mol}$

$\mathrm{R}=8.08 \mathrm{kcal} / \mathrm{mol}$

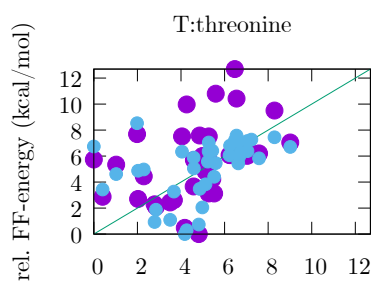

$\mathrm{M}=3.03 \mathrm{kcal} / \mathrm{mol}$

$\mathrm{R}=8.21 \mathrm{kcal} / \mathrm{mol}$

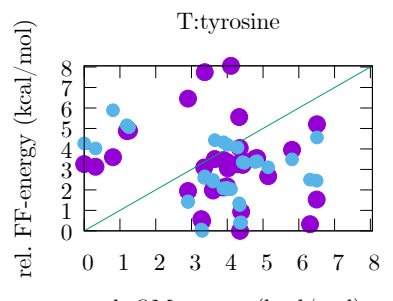

rel. QM-energy (kcal/mol)

$\mathrm{M}=6.35 \mathrm{kcal} / \mathrm{mol}$

$\mathrm{R}=6.49 \mathrm{kcal} / \mathrm{mol}$

Figure S2: "physical" conformers are compared with the respective QM conformers of the force fields (FF) in purple. Single point ReaxFF energies of the QM conformers are shown in blue. The energy values are relative to the respective most stable conformer. A linear curve is used as a guide to the eye. For every plot a metric of monotony $\mathrm{M}$ and an energetic resolution $\mathrm{R}$ is provided. 


\subsection{Prediction of charges}

Table S1: Atomic partial charges of Glycine

\begin{tabular}{c|c|c|c|c|c} 
Glycine & $\mathrm{C}$ & $\mathrm{C} 2$ & $\mathrm{G}$ & $\mathrm{T}$ & $\mathrm{QM}$ \\
\hline N1 & -0.534 & -0.589 & -0.514 & -0.566 & -0.557 \\
\hline C2 & -0.155 & -0.022 & -0.149 & -0.057 & -0.219 \\
\hline C3 & 0.612 & 0.638 & 0.565 & 0.254 & 0.123 \\
\hline O4 & -0.407 & -0.428 & -0.386 & -0.212 & -0.307 \\
\hline H5 & 0.218 & 0.223 & 0.209 & 0.233 & 0.248 \\
\hline H6 & 0.219 & 0.223 & 0.211 & 0.235 & 0.254 \\
\hline H7 & 0.120 & 0.084 & 0.121 & 0.077 & 0.180 \\
\hline H8 & 0.120 & 0.084 & 0.121 & 0.075 & 0.180 \\
\hline O9 & -0.541 & -0.557 & -0.524 & -0.385 & -0.200 \\
\hline H10 & 0.348 & 0.344 & 0.346 & 0.345 & 0.296 \\
\hline
\end{tabular}

Table S2: Atomic partial charges of Valine

\begin{tabular}{c|c|c|c|c|c} 
Valine & $\mathrm{C}$ & $\mathrm{C} 2$ & $\mathrm{G}$ & $\mathrm{T}$ & $\mathrm{QM}$ \\
\hline N1 & -0.542 & -0.589 & -0.518 & -0.567 & -0.527 \\
\hline C2 & -0.049 & 0.036 & -.047 & 0.017 & 0.025 \\
\hline C3 & 0.594 & 0.628 & 0.552 & 0.250 & -0.059 \\
\hline O4 & -0.555 & -0.569 & -0.538 & -0.393 & -0.192 \\
\hline H5 & 0.204 & 0.209 & 0.195 & 0.228 & 0.253 \\
\hline H6 & 0.211 & 0.212 & 0.203 & 0.245 & 0.246 \\
\hline H7 & 0.109 & 0.079 & 0.113 & 0.081 & 0.180 \\
\hline C8 & -0.086 & -0.091 & -0.103 & -0.096 & 0.068 \\
\hline C9 & -0.325 & -0.221 & -0.321 & -0.227 & -0.541 \\
\hline C10 & -0.306 & -0.202 & -0.299 & -0.219 & -0.492 \\
\hline H11 & 0.143 & 0.113 & 0.148 & 0.123 & 0.176 \\
\hline H12 & 0.108 & 0.078 & 0.107 & 0.064 & 0.138 \\
\hline H13 & 0.113 & 0.083 & 0.113 & 0.078 & 0.146 \\
\hline H14 & 0.114 & 0.081 & 0.113 & 0.068 & 0.135 \\
\hline H15 & 0.143 & 0.109 & 0.143 & 0.113 & 0.165 \\
\hline H16 & 0.108 & 0.076 & 0.108 & 0.055 & 0.115 \\
\hline H17 & 0.112 & 0.082 & 0.111 & 0.074 & 0.147 \\
\hline O18 & -0.435 & -0.453 & -0.415 & -0.233 & -0.275 \\
\hline H19 & 0.338 & 0.337 & 0.336 & 0.338 & 0.289 \\
\hline
\end{tabular}


Table S3: Atomic partial charges of Tryptophane

\begin{tabular}{c|c|c|c|c|c} 
Tryptophan & $\mathrm{C}$ & $\mathrm{C} 2$ & $\mathrm{G}$ & $\mathrm{T}$ & $\mathrm{QM}$ \\
\hline N1 & -0.543 & -0.589 & -0.519 & -0.577 & -0.592 \\
\hline C2 & -0.049 & 0.031 & -0.053 & 0.012 & 0.114 \\
\hline C3 & 0.607 & 0.634 & 0.565 & 0.259 & -0.122 \\
\hline O4 & -0.558 & -0.573 & -0.541 & -0.395 & -0.163 \\
\hline H5 & 0.235 & 0.227 & 0.227 & 0.281 & 0.287 \\
\hline H6 & 0.341 & 0.338 & 0.338 & 0.343 & 0.286 \\
\hline H7 & 0.101 & 0.072 & 0.104 & 0.069 & 0.178 \\
\hline C8 & -0.228 & -0.165 & -0.221 & -0.166 & -0.879 \\
\hline C9 & -0.009 & -0.018 & -0.011 & -0.017 & 0.584 \\
\hline C10 & 0.034 & 0.108 & 0.004 & 0.043 & -0.083 \\
\hline C11 & 0.002 & $-0.000(2)$ & 0.008 & $0.000(7)$ & 1.518 \\
\hline N12 & -0.392 & -0.462 & -0.338 & -0.414 & -0.139 \\
\hline C13 & 0.163 & 0.181 & 0.125 & 0.137 & -0.540 \\
\hline C14 & -0.129 & -0.097 & -0.129 & -0.103 & -1.061 \\
\hline C15 & -0.134 & -0.089 & -0.123 & -0.097 & -0.157 \\
\hline C16 & -0.102 & -0.077 & -0.107 & -0.087 & -0.171 \\
\hline C17 & -0.103 & -0.081 & -0.108 & -0.089 & -0.312 \\
\hline H18 & 0.105 & 0.073 & 0.105 & 0.065 & 0.144 \\
\hline H19 & 0.133 & 0.098 & 0.134 & 0.102 & 0.192 \\
\hline H20 & 0.135 & 0.108 & 0.141 & 0.103 & 0.149 \\
\hline H21 & 0.204 & 0.211 & 0.189 & 0.244 & 0.311 \\
\hline H22 & 0.103 & 0.077 & 0.105 & 0.065 & 0.111 \\
\hline H23 & 0.113 & 0.085 & 0.114 & 0.074 & 0.110 \\
\hline H24 & 0.109 & 0.086 & 0.113 & 0.081 & 0.122 \\
\hline H25 & 0.112 & 0.087 & 0.115 & 0.084 & 0.124 \\
\hline O26 & -0.447 & -0.468 & -0.427 & -0.242 & -0.266 \\
\hline H27 & 0.199 & 0.205 & 0.189 & 0.222 & 0.254 \\
\hline & & & & & \\
\hline
\end{tabular}


Table S4: Atomic partial charges of Alanine

\begin{tabular}{c|c|c|c|c|c} 
Alanine & $\mathrm{C}$ & $\mathrm{C} 2$ & $\mathrm{G}$ & $\mathrm{T}$ & $\mathrm{QM}$ \\
\hline $\mathrm{N}$ & -0.541 & -0.588 & -0.516 & -0.564 & -0.504 \\
\hline $\mathrm{C}$ & -0.047 & 0.028 & -0.055 & 0.007 & -0.189 \\
\hline $\mathrm{C}$ & 0.607 & 0.638 & 0.565 & 0.258 & 0.137 \\
\hline $\mathrm{O}$ & -0.548 & -0.563 & -0.532 & -0.390 & -0.183 \\
\hline $\mathrm{H}$ & 0.215 & 0.217 & 0.205 & 0.238 & 0.239 \\
\hline $\mathrm{H}$ & 0.211 & 0.217 & 0.203 & 0.227 & 0.249 \\
\hline $\mathrm{H}$ & 0.343 & 0.340 & 0.341 & 0.342 & 0.293 \\
\hline $\mathrm{H}$ & 0.115 & 0.083 & 0.120 & 0.082 & 0.179 \\
\hline $\mathrm{C}$ & -0.321 & -0.215 & -0.313 & -0.230 & -0.411 \\
\hline $\mathrm{H}$ & 0.110 & 0.076 & 0.108 & 0.052 & 0.142 \\
\hline $\mathrm{H}$ & 0.141 & 0.105 & 0.140 & 0.106 & 0.179 \\
\hline $\mathrm{H}$ & 0.135 & 0.100 & 0.134 & 0.094 & 0.159 \\
\hline $\mathrm{O}$ & -0.419 & -0.439 & -0.399 & -0.221 & -0.292 \\
\hline
\end{tabular}


Table S5: Atomic partial charges of Arginine

\begin{tabular}{c|c|c|c|c|c} 
Arginine & $\mathrm{C}$ & $\mathrm{C} 2$ & $\mathrm{G}$ & $\mathrm{T}$ & $\mathrm{QM}$ \\
\hline $\mathrm{N}$ & -0.489 & -0.539 & -0.462 & -0.522 & -0.398 \\
\hline $\mathrm{C}$ & -0.050 & 0.032 & -0.054 & 0.014 & -0.152 \\
\hline $\mathrm{C}$ & 0.581 & 0.615 & 0.540 & 0.237 & -0.055 \\
\hline $\mathrm{O}$ & -0.430 & -0.447 & -0.410 & -0.247 & -0.305 \\
\hline $\mathrm{H}$ & 0.238 & 0.241 & 0.229 & 0.271 & 0.241 \\
\hline $\mathrm{H}$ & 0.207 & 0.213 & 0.198 & 0.229 & 0.223 \\
\hline $\mathrm{H}$ & 0.113 & 0.079 & 0.116 & 0.080 & 0.158 \\
\hline $\mathrm{C}$ & -0.187 & -0.132 & -0.189 & -0.142 & 0.050 \\
\hline $\mathrm{C}$ & -0.212 & -0.155 & -0.214 & -0.159 & -0.482 \\
\hline $\mathrm{C}$ & -0.070 & 0.019 & -0.109 & -0.036 & -0.154 \\
\hline $\mathrm{N}$ & -0.437 & -0.511 & -0.359 & -0.425 & -0.266 \\
\hline $\mathrm{C}$ & 0.500 & 0.600 & 0.375 & 0.411 & 0.172 \\
\hline $\mathrm{N}$ & -0.503 & -0.544 & -0.436 & -0.496 & -0.640 \\
\hline $\mathrm{N}$ & -0.602 & -0.634 & -0.538 & -0.578 & -0.389 \\
\hline $\mathrm{H}$ & 0.136 & 0.101 & 0.139 & 0.108 & 0.135 \\
\hline $\mathrm{H}$ & 0.124 & 0.095 & 0.125 & 0.087 & 0.153 \\
\hline $\mathrm{H}$ & 0.107 & 0.078 & 0.108 & 0.062 & 0.139 \\
\hline $\mathrm{H}$ & 0.120 & 0.089 & 0.122 & 0.089 & 0.184 \\
\hline $\mathrm{H}$ & 0.099 & 0.074 & 0.104 & 0.054 & 0.152 \\
\hline $\mathrm{H}$ & 0.129 & 0.100 & 0.134 & 0.117 & 0.161 \\
\hline $\mathrm{H}$ & 0.197 & 0.204 & 0.181 & 0.221 & 0.243 \\
\hline $\mathrm{H}$ & 0.229 & 0.233 & 0.214 & 0.247 & 0.226 \\
\hline $\mathrm{H}$ & 0.220 & 0.223 & 0.207 & 0.222 & 0.247 \\
\hline $\mathrm{H}$ & 0.219 & 0.223 & 0.204 & 0.225 & 0.226 \\
\hline $\mathrm{O}$ & -0.573 & -0.590 & -0.556 & -0.416 & -0.307 \\
\hline $\mathrm{H}$ & 0.336 & 0.332 & 0.330 & 0.346 & 0.436 \\
\hline & & & & &
\end{tabular}


Table S6: Atomic partial charges of Asparagine

\begin{tabular}{c|c|c|c|c|c} 
Asparagine & $\mathrm{C}$ & $\mathrm{C} 2$ & $\mathrm{G}$ & $\mathrm{T}$ & $\mathrm{QM}$ \\
\hline $\mathrm{N}$ & -0.548 & -0.596 & -0.521 & -0.593 & -0.508 \\
\hline $\mathrm{C}$ & -0.057 & 0.018 & -0.063 & 0.006 & 0.099 \\
\hline $\mathrm{C}$ & 0.608 & 0.638 & 0.567 & 0.262 & -0.048 \\
\hline $\mathrm{O}$ & -0.438 & -0.459 & -0.418 & -0.252 & -0.302 \\
\hline $\mathrm{H}$ & 0.220 & 0.226 & 0.209 & 0.231 & 0.246 \\
\hline $\mathrm{H}$ & 0.282 & 0.282 & 0.273 & 0.320 & 0.272 \\
\hline $\mathrm{H}$ & 0.111 & 0.080 & 0.114 & 0.073 & 0.194 \\
\hline $\mathrm{C}$ & -0.275 & -0.179 & -0.245 & -0.167 & -0.379 \\
\hline $\mathrm{C}$ & 0.510 & 0.537 & 0.438 & 0.281 & 0.076 \\
\hline $\mathrm{O}$ & -0.437 & -0.456 & -0.413 & -0.295 & -0.369 \\
\hline $\mathrm{N}$ & -0.588 & -0.616 & -0.530 & -0.580 & -0.314 \\
\hline $\mathrm{H}$ & 0.129 & 0.092 & 0.127 & 0.074 & 0.187 \\
\hline $\mathrm{H}$ & 0.132 & 0.093 & 0.129 & 0.084 & 0.186 \\
\hline $\mathrm{H}$ & 0.268 & 0.267 & 0.252 & 0.300 & 0.268 \\
\hline $\mathrm{H}$ & 0.279 & 0.283 & 0.264 & 0.300 & 0.266 \\
\hline $\mathrm{O}$ & -0.545 & -0.560 & -0.529 & -0.393 & -0.166 \\
\hline $\mathrm{H}$ & 0.350 & 0.348 & 0.347 & 0.349 & 0.291 \\
\hline
\end{tabular}

Table S7: Atomic partial charges of Aspartic acid

\begin{tabular}{c|c|c|c|c|c} 
Aspartic acid & $\mathrm{C}$ & $\mathrm{C} 2$ & $\mathrm{G}$ & $\mathrm{T}$ & $\mathrm{QM}$ \\
\hline $\mathrm{N}$ & -0.545 & -0.593 & -0.519 & -0.586 & -0.486 \\
\hline $\mathrm{C}$ & -0.052 & 0.024 & -0.058 & -0.002 & 0.025 \\
\hline $\mathrm{C}$ & 0.594 & 0.627 & 0.551 & 0.243 & -0.051 \\
\hline $\mathrm{O}$ & -0.407 & -0.429 & -0.388 & -0.218 & -0.296 \\
\hline $\mathrm{H}$ & 0.259 & 0.264 & 0.249 & 0.285 & 0.271 \\
\hline $\mathrm{H}$ & 0.232 & 0.232 & 0.222 & 0.255 & 0.242 \\
\hline $\mathrm{H}$ & 0.152 & 0.117 & 0.155 & 0.126 & 0.209 \\
\hline $\mathrm{C}$ & -0.256 & -0.156 & -0.225 & -0.157 & -0.333 \\
\hline $\mathrm{C}$ & 0.587 & 0.604 & 0.533 & 0.231 & 0.155 \\
\hline $\mathrm{O}$ & -0.591 & -0.605 & -0.574 & -0.420 & -0.198 \\
\hline $\mathrm{O}$ & -0.461 & -0.479 & -0.440 & -0.281 & -0.317 \\
\hline $\mathrm{H}$ & 0.167 & 0.127 & 0.165 & 0.138 & 0.224 \\
\hline $\mathrm{H}$ & 0.148 & 0.108 & 0.145 & 0.108 & 0.189 \\
\hline $\mathrm{O}$ & -0.538 & -0.553 & -0.522 & -0.385 & -0.200 \\
\hline $\mathrm{H}$ & 0.347 & 0.346 & 0.344 & 0.340 & 0.294 \\
\hline $\mathrm{H}$ & 0.364 & 0.364 & 0.364 & 0.323 & 0.272 \\
\hline
\end{tabular}


Table S8: Atomic partial charges of Cysteine

\begin{tabular}{c|c|c|c} 
Cysteine & $\mathrm{C}$ & $\mathrm{C} 2$ & $\mathrm{QM}$ \\
\hline $\mathrm{N}$ & -0.541 & -0.588 & -0.476 \\
\hline $\mathrm{C}$ & -0.057 & 0.024 & 0.096 \\
\hline $\mathrm{C}$ & 0.602 & 0.634 & -0.059 \\
\hline $\mathrm{O}$ & -0.549 & -0.564 & -0.178 \\
\hline $\mathrm{H}$ & 0.240 & 0.241 & 0.258 \\
\hline $\mathrm{H}$ & 0.214 & 0.220 & 0.244 \\
\hline $\mathrm{H}$ & 0.110 & 0.078 & 0.188 \\
\hline $\mathrm{C}$ & -0.183 & -0.098 & -0.419 \\
\hline $\mathrm{S}$ & -0.192 & -0.212 & -0.149 \\
\hline $\mathrm{H}$ & 0.122 & 0.091 & 0.178 \\
\hline $\mathrm{H}$ & 0.142 & 0.109 & 0.214 \\
\hline $\mathrm{H}$ & 0.178 & 0.171 & 0.089 \\
\hline $\mathrm{O}$ & -0.429 & -0.449 & -0.277 \\
\hline $\mathrm{H}$ & 0.343 & 0.342 & 0.291 \\
\hline
\end{tabular}

Table S9: Atomic partial charges of Glutamic acid

\begin{tabular}{c|c|c|c|c|c} 
Glutamic acid & $\mathrm{C}$ & $\mathrm{C} 2$ & $\mathrm{G}$ & $\mathrm{T}$ & $\mathrm{QM}$ \\
\hline $\mathrm{N}$ & -0.553 & -0.601 & -0.527 & -0.607 & -0.582 \\
\hline $\mathrm{C}$ & -0.050 & 0.030 & -0.054 & 0.012 & 0.146 \\
\hline $\mathrm{C}$ & 0.600 & 0.630 & 0.558 & 0.254 & -0.083 \\
\hline $\mathrm{O}$ & -0.541 & -0.556 & -0.525 & -0.389 & -0.168 \\
\hline $\mathrm{H}$ & 0.223 & 0.230 & 0.213 & 0.237 & 0.243 \\
\hline $\mathrm{H}$ & 0.302 & 0.302 & 0.292 & 0.354 & 0.305 \\
\hline $\mathrm{H}$ & 0.110 & 0.081 & 0.114 & 0.074 & 0.187 \\
\hline $\mathrm{C}$ & -0.220 & -0.170 & -0.223 & -0.173 & -0.464 \\
\hline $\mathrm{C}$ & -0.236 & -0.142 & -0.211 & -0.148 & -0.107 \\
\hline $\mathrm{C}$ & 0.599 & 0.616 & 0.547 & 0.249 & 0.009 \\
\hline $\mathrm{O}$ & -0.495 & -0.511 & -0.473 & -0.324 & -0.334 \\
\hline $\mathrm{O}$ & -0.597 & -0.609 & -0.580 & -0.421 & -0.177 \\
\hline $\mathrm{H}$ & 0.131 & 0.098 & 0.131 & 0.089 & 0.166 \\
\hline $\mathrm{H}$ & 0.130 & 0.097 & 0.130 & 0.089 & 0.179 \\
\hline $\mathrm{H}$ & 0.140 & 0.107 & 0.138 & 0.107 & 0.184 \\
\hline $\mathrm{H}$ & 0.168 & 0.129 & 0.167 & 0.150 & 0.208 \\
\hline $\mathrm{O}$ & -0.423 & -0.442 & -0.404 & -0.228 & -0.269 \\
\hline $\mathrm{H}$ & 0.353 & 0.351 & 0.350 & 0.358 & 0.288 \\
\hline $\mathrm{H}$ & 0.358 & 0.358 & 0.357 & 0.319 & 0.269 \\
\hline
\end{tabular}


Table S10: Atomic partial charges of Glutamine

\begin{tabular}{c|c|c|c|c|c} 
Glutamine & $\mathrm{C}$ & $\mathrm{C} 2$ & $\mathrm{G}$ & $\mathrm{T}$ & $\mathrm{QM}$ \\
\hline $\mathrm{N}$ & -0.549 & -0.594 & -0.526 & -0.568 & -0.496 \\
\hline $\mathrm{C}$ & -0.056 & 0.024 & -0.057 & 0.007 & -0.160 \\
\hline $\mathrm{C}$ & 0.603 & 0.631 & 0.559 & 0.259 & 0.020 \\
\hline $\mathrm{O}$ & -0.547 & -0.561 & -0.531 & -0.390 & -0.172 \\
\hline $\mathrm{H}$ & 0.343 & 0.341 & 0.341 & 0.341 & 0.301 \\
\hline $\mathrm{H}$ & 0.216 & 0.222 & 0.208 & 0.227 & 0.254 \\
\hline $\mathrm{H}$ & 0.122 & 0.088 & 0.127 & 0.086 & 0.197 \\
\hline $\mathrm{C}$ & -0.218 & -0.167 & -0.220 & -0.166 & -0.194 \\
\hline $\mathrm{C}$ & -0.249 & -0.161 & -0.225 & -0.163 & -0.260 \\
\hline $\mathrm{C}$ & 0.498 & 0.527 & 0.427 & 0.271 & 0.059 \\
\hline $\mathrm{O}$ & -0.418 & -0.436 & -0.394 & -0.257 & -0.356 \\
\hline $\mathrm{N}$ & -0.596 & -0.623 & -0.540 & -0.592 & -0.378 \\
\hline $\mathrm{H}$ & 0.126 & 0.094 & 0.126 & 0.084 & 0.167 \\
\hline $\mathrm{H}$ & 0.133 & 0.101 & 0.134 & 0.086 & 0.174 \\
\hline $\mathrm{H}$ & 0.150 & 0.113 & 0.149 & 0.115 & 0.176 \\
\hline $\mathrm{H}$ & 0.133 & 0.100 & 0.132 & 0.091 & 0.185 \\
\hline $\mathrm{H}$ & 0.271 & 0.275 & 0.256 & 0.291 & 0.262 \\
\hline $\mathrm{H}$ & 0.291 & 0.290 & 0.275 & 0.332 & 0.299 \\
\hline $\mathrm{O}$ & -0.464 & -0.483 & -0.443 & -0.285 & -0.321 \\
\hline $\mathrm{H}$ & 0.214 & 0.218 & 0.204 & 0.231 & 0.243 \\
\hline
\end{tabular}


Table S11: Atomic partial charges of Histidine

\begin{tabular}{c|c|c|c|c|c} 
Histidine & $\mathrm{C}$ & $\mathrm{C} 2$ & $\mathrm{G}$ & $\mathrm{T}$ & $\mathrm{QM}$ \\
\hline $\mathrm{N}$ & -0.544 & -0.591 & -0.519 & -0.573 & -0.555 \\
\hline $\mathrm{C}$ & -0.050 & 0.028 & -0.055 & 0.009 & 0.238 \\
\hline $\mathrm{C}$ & 0.614 & 0.645 & 0.570 & 0.263 & -0.120 \\
\hline $\mathrm{O}$ & -0.449 & -0.469 & -0.429 & -0.259 & -0.292 \\
\hline $\mathrm{H}$ & 0.226 & 0.220 & 0.219 & 0.263 & 0.275 \\
\hline $\mathrm{H}$ & 0.342 & 0.340 & 0.340 & 0.344 & 0.289 \\
\hline $\mathrm{H}$ & 0.106 & 0.076 & 0.109 & 0.076 & 0.187 \\
\hline $\mathrm{C}$ & -0.246 & -0.175 & -0.230 & -0.174 & -1.096 \\
\hline $\mathrm{C}$ & 0.133 & 0.150 & 0.091 & 0.107 & 0.614 \\
\hline $\mathrm{N}$ & -0.391 & -0.479 & -0.319 & -0.436 & -0.174 \\
\hline $\mathrm{C}$ & -0.028 & 0.066 & -0.052 & 0.030 & -0.334 \\
\hline $\mathrm{C}$ & 0.150 & 0.277 & 0.075 & 0.182 & 0.096 \\
\hline $\mathrm{N}$ & -0.218 & -0.312 & -0.162 & -0.259 & -0.152 \\
\hline $\mathrm{H}$ & 0.100 & 0.062 & 0.101 & 0.048 & 0.168 \\
\hline $\mathrm{H}$ & 0.138 & 0.104 & 0.137 & 0.102 & 0.203 \\
\hline $\mathrm{H}$ & 0.260 & 0.266 & 0.243 & 0.316 & 0.313 \\
\hline $\mathrm{H}$ & 0.105 & 0.074 & 0.110 & 0.064 & 0.119 \\
\hline $\mathrm{H}$ & 0.106 & 0.080 & 0.116 & 0.067 & 0.129 \\
\hline $\mathrm{O}$ & -0.557 & -0.572 & -0.540 & -0.395 & -0.161 \\
\hline $\mathrm{H}$ & 0.204 & 0.209 & 0.194 & 0.224 & 0.251 \\
\hline
\end{tabular}


Table S12: Atomic partial charges of Isoleucine

\begin{tabular}{c|c|c|c|c|c} 
Isoleucine & $\mathrm{C}$ & $\mathrm{C} 2$ & $\mathrm{G}$ & $\mathrm{T}$ & $\mathrm{QM}$ \\
\hline $\mathrm{N}$ & -0.543 & -0.589 & -0.520 & -0.566 & -0.510 \\
\hline $\mathrm{C}$ & -0.055 & 0.032 & -0.053 & 0.012 & -0.118 \\
\hline $\mathrm{C}$ & 0.590 & 0.625 & 0.548 & 0.247 & -0.072 \\
\hline $\mathrm{O}$ & -0.557 & -0.571 & -0.541 & -0.395 & -0.176 \\
\hline $\mathrm{H}$ & 0.202 & 0.208 & 0.193 & 0.226 & 0.255 \\
\hline $\mathrm{H}$ & 0.336 & 0.335 & 0.334 & 0.336 & 0.285 \\
\hline $\mathrm{H}$ & 0.109 & 0.078 & 0.112 & 0.081 & 0.185 \\
\hline $\mathrm{C}$ & -0.089 & -0.087 & -0.101 & -0.093 & 0.154 \\
\hline $\mathrm{C}$ & -0.185 & -0.137 & -0.191 & -0.152 & -0.107 \\
\hline $\mathrm{C}$ & -0.324 & -0.221 & -0.319 & -0.224 & -0.599 \\
\hline $\mathrm{C}$ & -0.320 & -0.217 & -0.318 & -0.230 & -0.572 \\
\hline $\mathrm{H}$ & 0.143 & 0.112 & 0.148 & 0.125 & 0.175 \\
\hline $\mathrm{H}$ & 0.141 & 0.111 & 0.145 & 0.122 & 0.161 \\
\hline $\mathrm{H}$ & 0.108 & 0.078 & 0.110 & 0.064 & 0.112 \\
\hline $\mathrm{H}$ & 0.111 & 0.079 & 0.111 & 0.067 & 0.143 \\
\hline $\mathrm{H}$ & 0.105 & 0.075 & 0.105 & 0.062 & 0.137 \\
\hline $\mathrm{H}$ & 0.112 & 0.080 & 0.111 & 0.078 & 0.145 \\
\hline $\mathrm{H}$ & 0.125 & 0.094 & 0.125 & 0.088 & 0.146 \\
\hline $\mathrm{H}$ & 0.111 & 0.082 & 0.110 & 0.071 & 0.144 \\
\hline $\mathrm{H}$ & 0.112 & 0.080 & 0.112 & 0.079 & 0.127 \\
\hline $\mathrm{O}$ & -0.438 & -0.456 & -0.418 & -0.235 & -0.263 \\
\hline $\mathrm{H}$ & 0.206 & 0.208 & 0.198 & 0.238 & 0.247 \\
\hline & & & & &
\end{tabular}


Table S13: Atomic partial charges of Leucine

\begin{tabular}{c|c|c|c|c|c} 
Leucine & $\mathrm{C}$ & $\mathrm{C} 2$ & $\mathrm{G}$ & $\mathrm{T}$ & $\mathrm{QM}$ \\
\hline $\mathrm{N}$ & -0.545 & -0.590 & -0.522 & -0.566 & -0.502 \\
\hline $\mathrm{C}$ & -0.051 & 0.030 & -0.054 & 0.007 & -0.078 \\
\hline $\mathrm{C}$ & 0.597 & 0.632 & 0.554 & 0.251 & -0.021 \\
\hline $\mathrm{O}$ & -0.555 & -0.569 & -0.539 & -0.393 & -0.200 \\
\hline $\mathrm{H}$ & 0.207 & 0.210 & 0.199 & 0.234 & 0.251 \\
\hline $\mathrm{H}$ & 0.338 & 0.337 & 0.336 & 0.338 & 0.292 \\
\hline $\mathrm{H}$ & 0.112 & 0.080 & 0.116 & 0.089 & 0.186 \\
\hline $\mathrm{C}$ & -0.207 & -0.146 & -0.202 & -0.158 & -0.351 \\
\hline $\mathrm{C}$ & -0.078 & -0.088 & -0.103 & -0.092 & 0.253 \\
\hline $\mathrm{C}$ & -0.324 & -0.216 & -0.318 & -0.225 & -0.565 \\
\hline $\mathrm{C}$ & -0.318 & -0.214 & -0.312 & -0.221 & -0.528 \\
\hline $\mathrm{H}$ & 0.145 & 0.111 & 0.146 & 0.121 & 0.189 \\
\hline $\mathrm{H}$ & 0.119 & 0.087 & 0.121 & 0.084 & 0.149 \\
\hline $\mathrm{H}$ & 0.113 & 0.087 & 0.119 & 0.083 & 0.122 \\
\hline $\mathrm{H}$ & 0.120 & 0.088 & 0.119 & 0.084 & 0.145 \\
\hline $\mathrm{H}$ & 0.108 & 0.079 & 0.108 & 0.067 & 0.136 \\
\hline $\mathrm{H}$ & 0.114 & 0.084 & 0.114 & 0.078 & 0.139 \\
\hline $\mathrm{H}$ & 0.112 & 0.082 & 0.112 & 0.075 & 0.139 \\
\hline $\mathrm{H}$ & 0.106 & 0.075 & 0.105 & 0.061 & 0.125 \\
\hline $\mathrm{H}$ & 0.121 & 0.088 & 0.121 & 0.087 & 0.151 \\
\hline $\mathrm{O}$ & -0.434 & -0.453 & -0.415 & -0.231 & -0.289 \\
\hline $\mathrm{H}$ & 0.201 & 0.208 & 0.193 & 0.225 & 0.255 \\
\hline & & & & &
\end{tabular}


Table S14: Atomic partial charges of Lysine

\begin{tabular}{c|c|c|c|c|c} 
Lysine & $\mathrm{C}$ & $\mathrm{C} 2$ & $\mathrm{G}$ & $\mathrm{T}$ & $\mathrm{QM}$ \\
\hline $\mathrm{N}$ & -0.547 & -0.594 & -0.522 & -0.587 & -0.585 \\
\hline $\mathrm{C}$ & -0.050 & 0.033 & -0.053 & 0.012 & -0.096 \\
\hline $\mathrm{C}$ & 0.595 & 0.629 & 0.552 & 0.252 & -0.079 \\
\hline $\mathrm{O}$ & -0.557 & -0.572 & -0.541 & -0.397 & -0.160 \\
\hline $\mathrm{H}$ & 0.252 & 0.255 & 0.241 & 0.314 & 0.361 \\
\hline $\mathrm{H}$ & 0.337 & 0.336 & 0.335 & 0.341 & 0.280 \\
\hline $\mathrm{H}$ & 0.106 & 0.074 & 0.110 & 0.074 & 0.179 \\
\hline $\mathrm{C}$ & -0.199 & -0.143 & -0.199 & -0.157 & -0.162 \\
\hline $\mathrm{C}$ & -0.191 & -0.142 & -0.200 & -0.152 & -0.233 \\
\hline $\mathrm{C}$ & -0.211 & -0.152 & -0.212 & -0.150 & -0.302 \\
\hline $\mathrm{C}$ & -0.083 & -0.000 & -0.110 & -0.043 & -0.310 \\
\hline $\mathrm{N}$ & -0.494 & -0.538 & -0.463 & -0.522 & -0.529 \\
\hline $\mathrm{H}$ & 0.128 & 0.096 & 0.130 & 0.100 & 0.186 \\
\hline $\mathrm{H}$ & 0.134 & 0.099 & 0.136 & 0.107 & 0.143 \\
\hline $\mathrm{H}$ & 0.126 & 0.096 & 0.128 & 0.087 & 0.155 \\
\hline $\mathrm{H}$ & 0.100 & 0.074 & 0.102 & 0.066 & 0.152 \\
\hline $\mathrm{H}$ & 0.105 & 0.078 & 0.108 & 0.067 & 0.146 \\
\hline $\mathrm{H}$ & 0.100 & 0.071 & 0.103 & 0.067 & 0.152 \\
\hline $\mathrm{H}$ & 0.089 & 0.065 & 0.095 & 0.046 & 0.141 \\
\hline $\mathrm{H}$ & 0.109 & 0.083 & 0.114 & 0.075 & 0.146 \\
\hline $\mathrm{H}$ & 0.196 & 0.201 & 0.188 & 0.220 & 0.221 \\
\hline $\mathrm{H}$ & 0.191 & 0.199 & 0.182 & 0.204 & 0.239 \\
\hline $\mathrm{O}$ & -0.438 & -0.456 & -0.418 & -0.236 & -0.287 \\
\hline $\mathrm{H}$ & 0.201 & 0.208 & 0.192 & 0.216 & 0.242 \\
\hline & & & & &
\end{tabular}


Table S15: Atomic partial charges of Methionine

\begin{tabular}{c|c|c|c} 
Methionine & $\mathrm{C}$ & $\mathrm{C} 2$ & $\mathrm{QM}$ \\
\hline $\mathrm{N}$ & -0.546 & -0.591 & -0.469 \\
\hline $\mathrm{C}$ & -0.038 & 0.041 & -0.198 \\
\hline $\mathrm{C}$ & 0.592 & 0.628 & -0.013 \\
\hline $\mathrm{O}$ & -0.433 & -0.452 & -0.292 \\
\hline $\mathrm{H}$ & 0.217 & 0.222 & 0.258 \\
\hline $\mathrm{H}$ & 0.211 & 0.213 & 0.247 \\
\hline $\mathrm{H}$ & 0.132 & 0.098 & 0.220 \\
\hline $\mathrm{C}$ & -0.215 & -0.159 & -0.348 \\
\hline $\mathrm{C}$ & -0.170 & -0.096 & -0.407 \\
\hline $\mathrm{S}$ & -0.126 & -0.167 & 0.383 \\
\hline $\mathrm{C}$ & -0.270 & -0.147 & -0.787 \\
\hline $\mathrm{H}$ & 0.141 & 0.106 & 0.201 \\
\hline $\mathrm{H}$ & 0.114 & 0.083 & 0.160 \\
\hline $\mathrm{H}$ & 0.112 & 0.084 & 0.193 \\
\hline $\mathrm{H}$ & 0.119 & 0.092 & 0.175 \\
\hline $\mathrm{H}$ & 0.128 & 0.097 & 0.182 \\
\hline $\mathrm{H}$ & 0.127 & 0.092 & 0.195 \\
\hline $\mathrm{H}$ & 0.118 & 0.085 & 0.182 \\
\hline $\mathrm{O}$ & -0.551 & -0.566 & -0.198 \\
\hline $\mathrm{H}$ & 0.338 & 0.337 & 0.290 \\
\hline
\end{tabular}


Table S16: Atomic partial charges of Phenyalanine

\begin{tabular}{c|c|c|c|c|c} 
Phenylalanine & $\mathrm{C}$ & $\mathrm{C} 2$ & $\mathrm{G}$ & $\mathrm{T}$ & $\mathrm{QM}$ \\
\hline $\mathrm{N}$ & -0.542 & -0.588 & -0.518 & -0.574 & -0.576 \\
\hline $\mathrm{C}$ & -0.047 & 0.032 & -0.051 & 0.013 & 0.053 \\
\hline $\mathrm{C}$ & 0.606 & 0.635 & 0.564 & 0.259 & -0.118 \\
\hline $\mathrm{O}$ & -0.447 & -0.465 & -0.427 & -0.242 & -0.258 \\
\hline $\mathrm{H}$ & 0.200 & 0.206 & 0.190 & 0.222 & 0.252 \\
\hline $\mathrm{H}$ & 0.230 & 0.224 & 0.222 & 0.273 & 0.279 \\
\hline $\mathrm{H}$ & 0.103 & 0.074 & 0.106 & 0.072 & 0.182 \\
\hline $\mathrm{C}$ & -0.231 & -0.159 & -0.223 & -0.163 & -0.543 \\
\hline $\mathrm{C}$ & 0.023 & -0.003 & 0.010 & -0.006 & 1.084 \\
\hline $\mathrm{C}$ & -0.083 & -0.050 & -0.079 & -0.073 & -0.409 \\
\hline $\mathrm{C}$ & -0.117 & -0.082 & -0.115 & -0.088 & -0.298 \\
\hline $\mathrm{C}$ & -0.099 & -0.071 & -0.103 & -0.083 & -0.374 \\
\hline $\mathrm{C}$ & -0.102 & -0.076 & -0.107 & -0.085 & -0.272 \\
\hline $\mathrm{C}$ & -0.098 & -0.072 & -0.103 & -0.080 & -0.124 \\
\hline $\mathrm{H}$ & 0.103 & 0.072 & 0.103 & 0.062 & 0.142 \\
\hline $\mathrm{H}$ & 0.136 & 0.103 & 0.137 & 0.107 & 0.191 \\
\hline $\mathrm{H}$ & 0.149 & 0.121 & 0.152 & 0.130 & 0.154 \\
\hline $\mathrm{H}$ & 0.101 & 0.076 & 0.104 & 0.063 & 0.116 \\
\hline $\mathrm{H}$ & 0.113 & 0.088 & 0.116 & 0.083 & 0.133 \\
\hline $\mathrm{H}$ & 0.109 & 0.085 & 0.112 & 0.080 & 0.128 \\
\hline $\mathrm{H}$ & 0.109 & 0.085 & 0.112 & 0.081 & 0.128 \\
\hline $\mathrm{O}$ & -0.558 & -0.572 & -0.541 & -0.394 & -0.159 \\
\hline $\mathrm{H}$ & 0.341 & 0.338 & 0.339 & 0.343 & 0.286 \\
\hline
\end{tabular}


Table S17: Atomic partial charges of Proline

\begin{tabular}{c|c|c|c|c|c} 
Proline & $\mathrm{C}$ & $\mathrm{C} 2$ & $\mathrm{G}$ & $\mathrm{T}$ & $\mathrm{QM}$ \\
\hline $\mathrm{N}$ & -0.378 & -0.464 & -0.342 & -0.423 & -0.375 \\
\hline $\mathrm{C}$ & -0.064 & 0.021 & -0.067 & 0.011 & -0.109 \\
\hline $\mathrm{C}$ & 0.600 & 0.632 & 0.557 & 0.252 & 0.055 \\
\hline $\mathrm{O}$ & -0.427 & -0.446 & -0.407 & -0.225 & -0.285 \\
\hline $\mathrm{H}$ & 0.186 & 0.197 & 0.175 & 0.223 & 0.255 \\
\hline $\mathrm{H}$ & 0.106 & 0.075 & 0.109 & 0.071 & 0.179 \\
\hline $\mathrm{C}$ & -0.194 & -0.142 & -0.196 & -0.145 & -0.233 \\
\hline $\mathrm{C}$ & -0.196 & -0.142 & -0.199 & -0.146 & -0.304 \\
\hline $\mathrm{C}$ & -0.094 & -0.005 & -0.122 & -0.045 & -0.269 \\
\hline $\mathrm{H}$ & 0.108 & 0.077 & 0.109 & 0.071 & 0.146 \\
\hline $\mathrm{H}$ & 0.136 & 0.105 & 0.138 & 0.107 & 0.186 \\
\hline $\mathrm{H}$ & 0.134 & 0.103 & 0.137 & 0.108 & 0.172 \\
\hline $\mathrm{H}$ & 0.099 & 0.070 & 0.101 & 0.060 & 0.162 \\
\hline $\mathrm{H}$ & 0.107 & 0.083 & 0.111 & 0.069 & 0.163 \\
\hline $\mathrm{H}$ & 0.098 & 0.071 & 0.104 & 0.062 & 0.139 \\
\hline $\mathrm{O}$ & -0.552 & -0.566 & -0.535 & -0.389 & -0.177 \\
\hline $\mathrm{H}$ & 0.330 & 0.329 & 0.327 & 0.339 & 0.294 \\
\hline
\end{tabular}

Table S18: Atomic partial charges of Serine

\begin{tabular}{c|c|c|c|c|c} 
Serine & $\mathrm{C}$ & $\mathrm{C} 2$ & $\mathrm{G}$ & $\mathrm{T}$ & $\mathrm{QM}$ \\
\hline $\mathrm{N}$ & -0.539 & -0.590 & -0.515 & -0.582 & -0.484 \\
\hline $\mathrm{C}$ & -0.076 & 0.017 & -0.072 & 0.009 & -0.078 \\
\hline $\mathrm{C}$ & 0.608 & 0.638 & 0.564 & 0.254 & 0.056 \\
\hline $\mathrm{O}$ & -0.431 & -0.452 & -0.412 & -0.236 & -0.304 \\
\hline $\mathrm{H}$ & 0.214 & 0.219 & 0.204 & 0.231 & 0.244 \\
\hline $\mathrm{H}$ & 0.264 & 0.267 & 0.255 & 0.296 & 0.267 \\
\hline $\mathrm{H}$ & 0.099 & 0.065 & 0.102 & 0.066 & 0.182 \\
\hline $\mathrm{C}$ & 0.003 & 0.082 & -0.008 & -0.062 & -0.255 \\
\hline $\mathrm{O}$ & -0.519 & -0.548 & -0.515 & -0.415 & -0.309 \\
\hline $\mathrm{H}$ & 0.123 & 0.096 & 0.125 & 0.091 & 0.154 \\
\hline $\mathrm{H}$ & 0.122 & 0.093 & 0.125 & 0.092 & 0.169 \\
\hline $\mathrm{H}$ & 0.332 & 0.328 & 0.333 & 0.305 & 0.257 \\
\hline $\mathrm{O}$ & -0.546 & -0.560 & -0.530 & -0.391 & -0.191 \\
\hline $\mathrm{H}$ & 0.347 & 0.345 & 0.344 & 0.343 & 0.293 \\
\hline
\end{tabular}


Table S19: Atomic partial charges of Threonine

\begin{tabular}{c|c|c|c|c|c} 
Threonine & $\mathrm{C}$ & $\mathrm{C} 2$ & $\mathrm{G}$ & $\mathrm{T}$ & $\mathrm{QM}$ \\
\hline $\mathrm{N}$ & -0.539 & -0.591 & -0.516 & -0.582 & -0.500 \\
\hline $\mathrm{C}$ & -0.072 & 0.025 & -0.064 & 0.016 & -0.037 \\
\hline $\mathrm{C}$ & 0.599 & 0.633 & 0.556 & 0.247 & 0.004 \\
\hline $\mathrm{O}$ & -0.549 & -0.564 & -0.533 & -0.392 & -0.195 \\
\hline $\mathrm{H}$ & 0.208 & 0.213 & 0.198 & 0.230 & 0.248 \\
\hline $\mathrm{H}$ & 0.259 & 0.262 & 0.250 & 0.294 & 0.278 \\
\hline $\mathrm{H}$ & 0.099 & 0.063 & 0.101 & 0.071 & 0.182 \\
\hline $\mathrm{C}$ & 0.113 & 0.136 & 0.090 & 0.008 & -0.093 \\
\hline $\mathrm{O}$ & -0.526 & -0.552 & -0.519 & -0.411 & -0.281 \\
\hline $\mathrm{C}$ & -0.313 & -0.197 & -0.302 & -0.214 & -0.480 \\
\hline $\mathrm{H}$ & 0.122 & 0.096 & 0.129 & 0.103 & 0.173 \\
\hline $\mathrm{H}$ & 0.324 & 0.322 & 0.325 & 0.295 & 0.259 \\
\hline $\mathrm{H}$ & 0.095 & 0.059 & 0.093 & 0.049 & 0.138 \\
\hline $\mathrm{H}$ & 0.139 & 0.107 & 0.137 & 0.101 & 0.163 \\
\hline $\mathrm{H}$ & 0.136 & 0.102 & 0.134 & 0.085 & 0.147 \\
\hline $\mathrm{O}$ & -0.439 & -0.459 & -0.420 & -0.242 & -0.300 \\
\hline $\mathrm{H}$ & 0.344 & 0.343 & 0.341 & 0.340 & 0.292 \\
\hline
\end{tabular}


Table S20: Atomic partial charges of Threonine

\begin{tabular}{c|c|c|c|c|c} 
Tyrosine & $\mathrm{C}$ & $\mathrm{C} 2$ & $\mathrm{G}$ & $\mathrm{T}$ & $\mathrm{QM}$ \\
\hline $\mathrm{N}$ & -0.543 & -0.589 & -0.518 & -0.575 & -0.578 \\
\hline $\mathrm{C}$ & -0.049 & 0.030 & -0.054 & 0.010 & 0.073 \\
\hline $\mathrm{C}$ & 0.605 & 0.634 & 0.563 & 0.258 & -0.122 \\
\hline $\mathrm{O}$ & -0.552 & -0.567 & -0.536 & -0.392 & -0.160 \\
\hline $\mathrm{H}$ & 0.231 & 0.225 & 0.223 & 0.273 & 0.278 \\
\hline $\mathrm{H}$ & 0.205 & 0.211 & 0.195 & 0.225 & 0.251 \\
\hline $\mathrm{H}$ & 0.108 & 0.079 & 0.111 & 0.076 & 0.181 \\
\hline $\mathrm{C}$ & -0.229 & -0.158 & -0.221 & -0.163 & -0.548 \\
\hline $\mathrm{C}$ & 0.014 & -0.013 & 0.000 & -0.011 & 1.336 \\
\hline $\mathrm{C}$ & -0.094 & -0.068 & -0.095 & -0.082 & -0.754 \\
\hline $\mathrm{C}$ & -0.139 & -0.107 & -0.139 & -0.104 & -0.650 \\
\hline $\mathrm{C}$ & -0.156 & -0.102 & -0.143 & -0.096 & -0.020 \\
\hline $\mathrm{C}$ & -0.108 & -0.059 & -0.098 & -0.074 & 0.243 \\
\hline $\mathrm{C}$ & 0.274 & 0.266 & 0.246 & 0.118 & -0.439 \\
\hline $\mathrm{O}$ & -0.539 & -0.553 & -0.529 & -0.393 & -0.259 \\
\hline $\mathrm{H}$ & 0.107 & 0.075 & 0.106 & 0.063 & 0.141 \\
\hline $\mathrm{H}$ & 0.139 & 0.106 & 0.140 & 0.109 & 0.189 \\
\hline $\mathrm{H}$ & 0.154 & 0.125 & 0.156 & 0.133 & 0.156 \\
\hline $\mathrm{H}$ & 0.107 & 0.080 & 0.109 & 0.064 & 0.119 \\
\hline $\mathrm{H}$ & 0.115 & 0.086 & 0.115 & 0.069 & 0.112 \\
\hline $\mathrm{H}$ & 0.147 & 0.119 & 0.147 & 0.119 & 0.131 \\
\hline $\mathrm{H}$ & 0.300 & 0.297 & 0.300 & 0.266 & 0.249 \\
\hline $\mathrm{O}$ & -0.440 & -0.458 & -0.420 & -0.238 & -0.259 \\
\hline $\mathrm{H}$ & 0.344 & 0.341 & 0.342 & 0.346 & 0.287 \\
\hline & & & & &
\end{tabular}

